TRANSACTIONS OF THE

AMERICAN MATHEMATICAL SOCIETY

Volume 353, Number 8, Pages 3267-3305

S 0002-9947(00)02675-1

Article electronically published on September 21, 2000

\title{
THE SIEGEL MODULAR VARIETY OF DEGREE TWO AND LEVEL THREE
}

\author{
J. WILLIAM HOFFMAN AND STEVEN H. WEINTRAUB
}

\begin{abstract}
Let $\mathcal{A}_{2}(n)$ denote the quotient of the Siegel upper half space of degree two by $\Gamma_{2}(n)$, the principal congruence subgroup of level $n$ in $\mathbf{S p}(4, \mathbf{Z})$. $\mathcal{A}_{2}(n)$ is the moduli space of principally polarized abelian varieties of dimension two with a level $n$ structure, and has a compactification $\mathcal{A}_{2}(n)^{*}$ first constructed by Igusa. When $n \geq 3$ this is a smooth projective algebraic variety of dimension three.

In this work we analyze the topology of $\mathcal{A}_{2}(3)^{*}$ and the open subset $\mathcal{A}_{2}(3)$. In this way we obtain the rational cohomology ring of $\Gamma_{2}(3)$. The key is that one has an explicit description of $\mathcal{A}_{2}(3)^{*}$ : it is the resolution of the 45 nodes on a projective quartic threefold whose equation was first written down about 100 years ago by $\mathrm{H}$. Burkhardt. We are able to compute the zeta function of this variety reduced modulo certain primes.
\end{abstract}

\section{IntRoduCtion}

1.1. Let $\mathfrak{S}_{d}$ be the Siegel upper half space of degree $d$ :

$$
\mathfrak{S}_{d}=\left\{\tau \in \mathbf{M}_{d}(\mathbf{C}):{ }^{t} \tau=\tau, \operatorname{Im}(\tau)>0\right\} .
$$

The group $\mathbf{S p}(2 d, \mathbf{R})$ operates on $\mathfrak{S}_{d}$ in the usual way,

$$
\gamma \cdot \tau=(A \tau+B)(C \tau+D)^{-1}, \quad \gamma=\left(\begin{array}{ll}
A & B \\
C & D
\end{array}\right) .
$$

This action factors through the projective group $\mathbf{P S p}(2 d, \mathbf{R})$. Let

$$
\Gamma \subset \mathbf{S p}(2 d, \mathbf{Q})
$$

be a subgroup commensurable with $\mathbf{S p}(2 d, \mathbf{Z})$. Then, Baily and Borel have shown that the quotient $\Gamma \backslash \mathfrak{S}_{d}$ admits the structure of a quasi-projective algebraic variety (of dimension $d(d+1) / 2$ ) [3]. In fact, this quotient is the set of $\mathbf{C}$-points of an algebraic variety defined over a number field; these are examples of Shimura varieties. These varieties are important from several points of view:

1. They are moduli spaces of abelian varieties with a principal polarization and a level structure.

2. Automorphic forms for the group $\mathbf{S p}_{2 d}$ and its metaplectic covering typically appear as sections of vector bundles over these spaces ([8]).

3. One has an isomorphism

$$
\mathrm{H}^{*}\left(\Gamma \backslash \mathfrak{S}_{d}, \mathbf{Q}\right) \simeq \mathrm{H}^{*}(\Gamma, \mathbf{Q})
$$

Received by the editors March 29, 1999.

2000 Mathematics Subject Classification. Primary 11F75, Secondary 11F46, 14G35, 14J30.

The first named author would like to thank Meijo University in Nagoya, Japan, for its generous hospitality. Part of this work was done while visiting there. 
with equality even over $\mathbf{Z}$ if $\Gamma$ is torsion-free, so this is a way of computing the cohomology of certain arithmetic groups.

Despite their fundamental importance, these spaces are not understood for $d \geq 2$. When $d=1$, these modular curves were studied, at least over $\mathbf{C}$, already in the $19^{\text {th }}$ century. For example, their homology groups were completely determined. As arithmetic schemes they have been studied by Shimura, Igusa, Deligne, and Rappoport, among others.

1.2. To proceed further, one must compactify these spaces. There are several compactifications.

1. The Borel-Serre compactification $\left(\Gamma \backslash \mathfrak{S}_{d}\right)^{b s}$. This is a manifold with corners [7. Using this, Borel and Serre give a formula for the virtual cohomological dimension:

$$
c=\operatorname{vcd}(\Gamma)=\operatorname{dim}_{\mathbf{R}}\left(\mathfrak{S}_{d}\right)-\operatorname{rank}\left(\mathbf{S p}_{2 d}\right)=d^{2},
$$

as well as the duality theorem:

$$
\mathrm{H}^{i}(\Gamma, \mathbf{Z}) \simeq \mathrm{H}_{c-i}(\Gamma, I),
$$

where $I \simeq \mathrm{H}^{c}(\Gamma, \mathbf{Z}[\Gamma])$ is the dualizing module.

2. The Satake compactification $\left(\Gamma \backslash \mathfrak{S}_{d}\right)^{s a}$. This is a projective algebraic variety [47]. However, its usefulness is limited by the severe nature of the singularities at infinity.

3. The toroidal compactifications of Mumford and his coworkers [2], 41], [26]. When $d=2$, this was first accomplished by Igusa [29], and Igusa's treatment of this case was one of the main motivations for Mumford's general theory. These compactifications depend on some choices, which in this case is that of a $\Gamma$-admissible rational polyhedral subdivision of the cone of positive $d \times d$ symmetric real matrices. When $d=1$ this is trivial, and when $d=2$ there is a canonical choice. For $d \geq 3$ one has existence but not uniqueness. We will refer to these compactifications generically as $\left(\Gamma \backslash \mathfrak{S}_{d}\right)^{m u}$, but in case $d=2$ with the canonical choice of subdivision we will call it the Igusa compactification. When $\Gamma$ is torsion-free it is a nonsingular projective algebraic variety and the boundary

$$
\partial\left(\left(\Gamma \backslash \mathfrak{S}_{d}\right)^{m u}\right)=\left(\Gamma \backslash \mathfrak{S}_{d}\right)^{m u}-\Gamma \backslash \mathfrak{S}_{d}
$$

is a divisor with normal crossings. The combinatorics of the boundary components is described by a finite geometry - the quotient by $\Gamma$ of the Tits building of rational parabolic subgroups of $\mathbf{S p}_{2 d}$. There is a morphism

$$
\left(\Gamma \backslash \mathfrak{S}_{d}\right)^{m u} \longrightarrow\left(\Gamma \backslash \mathfrak{S}_{d}\right)^{s a}
$$

which "blows down" the boundary components of the left-hand side to lowerdimensional varieties.

Faltings has generalized all of this to a theory of compactifications of the corresponding arithmetic schemes [16].

Set

$$
\Gamma_{d}(n)=\{\gamma \in \mathbf{S p}(2 d, \mathbf{Z}): \gamma \equiv \mathbf{1} \bmod n\},
$$

the principal congruence subgroup of degree $d$ and level $n$. We define

$$
\mathcal{A}_{d}(n)=\Gamma_{d}(n) \backslash \mathfrak{S}_{d}, \quad \mathcal{A}_{d}(n)^{*}=\left(\Gamma_{d}(n) \backslash \mathfrak{S}_{d}\right)^{m u} .
$$


When $d=2$ the right-hand equation is an unambiguous definition in that we always assume the canonical choice has been made for the decomposition of the cone of positive $2 \times 2$ symmetric matrices.

When $d=2$, all these are 3-dimensional; we refer to them generically as Siegel modular threefolds.

1.3. We focus on the topology of these spaces. In the following discussion, we take $d \geq 2$ and $\Gamma$ torsion-free. Known general results include the following:

1. $\pi_{1}\left(\left(\Gamma \backslash \mathfrak{S}_{d}\right)^{m u}\right)$ is finite (see [45]) and is 0 for the principal congruence subgroups (see [20]).

2. $\mathrm{H}_{1}(\Gamma, \mathbf{Z})=\Gamma /[\Gamma, \Gamma]$ is finite. This is true more generally for any lattice (= discrete subgroup of finite covolume) in $G(\mathbf{R})$, where $G$ is a semisimple group with R-rank at least 2 [30].

3. The Euler characteristic $\chi\left(\Gamma \backslash \mathfrak{S}_{d}\right)$ is known. This is a famous theorem of Siegel [52, which has been extended by Harder to $\Gamma \backslash G(\mathbf{R}) / K$ for Chevalley groups $G$ [18]. One also knows in principle how to compute the Euler characteristics $\chi\left(\left(\Gamma \backslash \mathfrak{S}_{d}\right)^{m u}\right)$, because in principle one can describe the boundary. In fact, for $d=2$ this can be done explicitly, at least for the principal congruence subgroups (see [24]).

4. For $n$ fixed, the $\Gamma_{d}(n)$ form an inductive system relative to natural inclusions $\mathbf{S p}(2 d, \mathbf{Z}) \rightarrow \mathbf{S p}(2(d+1), \mathbf{Z})$. A theorem of Borel [5] states that for $i$ fixed, as $d \rightarrow \infty \mathrm{H}^{i}\left(\Gamma_{d}(n), \mathbf{R}\right)$ has a stable value, equal to the Lie algebra cohomology $\mathrm{H}^{i}(\mathfrak{g}, \mathfrak{k}, \mathbf{R})$, where $\mathfrak{g}$ is the Lie algebra $\mathfrak{s p}_{2 d}(\mathbf{R})$ and $\mathfrak{k}=\mathfrak{u}_{d}$ is its maximal compact.

5. For $d=2$ general results have been obtained by Weissauer [56], [57, [58] and by Schwermer [50], and by Oda and Schwermer [42]. Weissauer's theorems relate various pieces of the cohomology of Siegel modular threefolds to automorphic representations. In this way he has found explicit formulas for the dimensions of the $(0,2)$ and $(0,3)$ part of the cohomology. He has also described the Picard group $(\otimes \mathbf{C})$ of these varieties in terms of automorphic forms.

6. Again for $d=2$, MacPherson and McConnell [39 have constructed an explicit compact polyhedron of dimension 4 , and a retraction of $\mathcal{A}_{2}(n)$ onto it. The description of the cell complex of dimension 4 arises from the Voronoi theory of quadratic forms in 4 variables, and the cells themselves are naturally indexed by certain configurations in the finite symplectic space $(\mathbf{Z} / n)^{4}$, in some cases generalizing classical projective geometry configurations. This gives a way of computing homology invariants of Siegel threefolds, but this has been of limited use so far, in part because of the huge number of cells required.

1.4. All this having been said, it must be emphasized that the homology groups of Siegel threefolds are known only in extremely few cases. In fact the only cases analyzed completely up till now are for the levels $n=1,2,4$. These results are due to Lee and Weintraub [32, [33, 34, 35, 36]. They determined $\mathrm{H}_{*}\left(\mathcal{A}_{2}(n)^{*}, \mathbf{Z}\right)$ for $n=1,2$ and $\mathrm{H}_{*}\left(\mathcal{A}_{2}(4)^{*}, \mathbf{Q}\right)$. From this they are able to compute the rational homology of $\Gamma_{2}(1)$ and $\Gamma_{2}(2)$. Recently we have also computed the rational homology of $\Gamma_{2}(4)$ as a corollary of the results of [35] (see [22]). The integral homology of $\mathbf{S p}_{4}(\mathbf{Z})$ was computed by Brownstein and Lee.

The main results of this paper are the determination of the cohomology for the case of level 3:

Theorem 1.1. a) $\mathrm{H}^{i}\left(\mathcal{A}_{2}(3)^{*}, \mathbf{Z}\right)$ is a free module of rank 1, 0, 61, 0, 61, 0, 1 for $i=0, \ldots, 6$, and 0 for all other values. 
b) $\mathrm{H}^{i}\left(\Gamma_{2}(3), \mathbf{Q}\right)$ has dimension 1, 0, 21, 139, 81 for $i=0, \ldots, 4$, and 0 for all other values.

c) $\operatorname{IH}^{i}\left(\mathcal{A}_{2}(3)^{\text {sa }}\right)$ has dimension 1, 0, 21, 0, 21, 0, 1 for $i=0, \ldots, 6$, and 0 for all other values.

In fact, our results are more precise. We can give explicit generators for these spaces - the cohomology is entirely represented by algebraic cycles. We can describe the (mixed) Hodge structures on these spaces. Also, all the intersection relations are determined, and therefore so is the ring structure on cohomology. Finally, the finite group $P \Gamma_{2}(1) / P \Gamma_{2}(3)$ operates as a group of symmetries on the whole situation, and we can decompose the cohomology spaces according to the characters of this group.

We remark that MacPherson and McConnell had found that $\mathrm{H}^{4}\left(\Gamma_{2}(3), \mathbf{Q}\right)$ was 81 dimensional, 39], and they had also computed $\mathrm{H}_{1}\left(\Gamma_{2}(3), \mathbf{Z}\right)$ (which is torsion).

1.5. The first step is to compute the Betti numbers of $\mathcal{A}_{2}(3)^{*}$, and this is accomplished by computing the zeta function of a variety $B$ defined over $\mathbf{Q}(\sqrt{-3})$ such that $B \otimes \mathbf{C}$ is isomorphic with $\mathcal{A}_{2}(3)^{*}$. Over 100 years ago Felix Klein initiated the study of the moduli spaces of genus 2 curves and the coverings defined by "Stufe". Two of his students, H. Burkhardt [9] and H. Maschke [40, took up the case where Stufe $=3$. Burkhardt managed to write down an explicit equation for this moduli space. The general idea is this: Consider the 9 thetanullwerte

$$
X_{\alpha, \beta}=\theta\left[\begin{array}{ll}
0 & 0 \\
\alpha & \beta
\end{array}\right](\tau, 0), \quad \alpha \in \frac{1}{3} \mathbf{Z} / \mathbf{Z} ; \beta \in \frac{1}{3} \mathbf{Z} / \mathbf{Z} .
$$

These 9 values have the property that as $\tau \rightarrow \gamma \cdot \tau$ with $\gamma \in P \Gamma_{2}(1)$ they undergo a linear transformation, which is the identity up to scalar multiples for $\gamma \in P \Gamma_{2}(3)$. In other words, we have a projective representation of the finite simple group of order 25920, $G=P \Gamma_{2}(1) / P \Gamma_{2}(3) \simeq \mathbf{P S p}\left(4, \mathbf{F}_{3}\right)$. This representation splits into two invariant subspaces of dimension 4 and 5 respectively, the spaces of

$$
\begin{aligned}
Z_{\alpha, \beta} & =\left(X_{\alpha, \beta}-X_{-\alpha,-\beta}\right) / 2, \\
Y_{\alpha, \beta} & =\left(X_{\alpha, \beta}+X_{-\alpha,-\beta}\right) / 2 .
\end{aligned}
$$

Maschke studied the action of $G$ on the $Z$ 's, Burkhardt studied the action on the $Y$ 's, and both managed to find the ring of $G$-invariant forms in their respective cases. Let

$$
-Y_{0}=Y_{0,0}, \quad 2 Y_{1}=Y_{\frac{1}{3}, 0}, \quad 2 Y_{2}=Y_{0, \frac{1}{3}}, \quad 2 Y_{3}=Y_{\frac{1}{3}, \frac{1}{3}}, \quad 2 Y_{4}=Y_{\frac{1}{3}, \frac{2}{3}} .
$$

Burkhardt found the invariant form of degree 4 :

$$
J_{4}=Y_{0}^{4}-Y_{0}\left(Y_{1}^{3}+Y_{2}^{3}+Y_{3}^{3}+Y_{4}^{3}\right)+3 Y_{1} Y_{2} Y_{3} Y_{4} .
$$

Theorem 1.2. Let $B_{0} \subset \mathbf{P}^{4}$ be the quartic hypersurface defined by $J_{4}=0$.

a) There is an isomorphism between a Zariski open subset of $\Gamma_{2}(3) \backslash \mathfrak{S}_{2}=\mathcal{A}_{2}(3)$ and a Zariski open subset of $B_{0}$.

b) Let $B$ be the variety obtained by resolving the 45 nodes on $B_{0}$. The the map in a) extends to an isomorphism with the Igusa compactification: $B \simeq \mathcal{A}_{2}(3)^{*}$.

In this form the theorem was first proved by van der Geer [54], who asserted something stronger, namely that these results were true for the corresponding schemes over $\mathbf{Z}[1 / 3, \varepsilon]$, where $\varepsilon$ is a primitive cube root of unity (the existence of a model of $\mathcal{A}_{2}(3)^{*}$ over that ring being a consequence of Faltings' theory [16]). However, [54] 
presents only an outline of an argument. A sketch of a proof of the above theorem can be found in [28], with complete details in [27].

The finite group $G$ was known in the $19^{\text {th }}$ century in another guise -it is the subgroup of index 2 in the group of symmetries of the configuration of 27 lines on a cubic surface. The dual nature of $G$ was noted as early as 1870 by C. Jordan, who devoted a whole chapter to it in his Traité des Substitutions, the first textbook on group theory. Studies of level 3 structures had been pursued even earlier than this, by Cayley and Clebsch among others, who obtained normal forms for genus 2 curves that exhibit the points of order 3 on the Jacobian. Also, in those days, the Galois viewpoint was important. As Klein realized, one could "solve" the equation (of degree 27) necessary to find the lines on a cubic surface by 3-division of genus 2 abelian functions in much the same way that one could "solve" the general quintic by 5 -division of elliptic functions. It is remarkable that these very same 27 lines play an important role in our work as well, when we compute the zeta function of $B$. For a beautiful account of all these matters, linking the classical with the modern, we recommend Bruce Hunt's monograph 27]. See also [11, 44.

One more general remark about Burkhardt's quartic. It is an example of a Janus-like variety in the terminology of [28] - that is, it admits the structure of a compactification of an arithmetic quotient of a bounded symmetric domain in 2 essentially different ways. It is also a quotient of the complex 3-ball, and it has a moduli interpretation as a parameter space of abelian 4 -folds with a certain type of PEL structure.

Theorem 1.3. Let $B$ be the resolution of the 45 nodes on the projective hypersurface defined by the equation $J_{4}=0$. Then for every prime power $q$ congruent to 1 modulo 3 the zeta function of $B$ regarded as a scheme over $\mathbf{F}_{q}$ is

$$
Z\left(B / \mathbf{F}_{q}, u\right)=\frac{1}{(1-u)(1-q u)^{61}\left(1-q^{2} u\right)^{61}\left(1-q^{3} u\right)} .
$$

In view of the above, the cohomological formula for the zeta function, and standard specialization and comparison theorems in étale cohomology, this gives part a) of theorem 1.2 (with Q-coefficients). Theorem 1.3 ought to be a corollary of the general results of Kottwitz [31] on the zeta functions of certain types of Shimura varieties, but it appears that the explicit evaluation of the terms involved in that theorem is difficult. This is a subject that ought to be studied more closely.

1.6. We will describe here some of the subvarieties that generate the cohomology. First, the boundary $\partial \mathcal{A}_{2}(3)^{*}$ is a union of 40 components $D(l)$ corresponding to the $\mathbf{Q}$-parabolic subgroups of corank 1 . The indexing set of the $l$ can be naturally identified with $\mathbf{P}^{3}\left(\mathbf{F}_{3}\right)$. More canonically, it is the quotient by \pm 1 of the nonzero vectors in $\mathbf{F}_{3}^{4}$. Each $D=D(l)$ is the elliptic modular surface [51] of level 3, and can be described concretely as follows: $D$ is the space obtained by blowing up $\mathbf{P}^{2}$ in the 9 base points of Hesse's pencil of elliptic curves

$$
X^{3}+Y^{3}+Z^{3}-3 \mu X Y Z=0 .
$$

The open subset $D^{0}$ of this where $\mu \neq 1, \varepsilon, \varepsilon^{2}$ fibers naturally over the $\mu$-line, which is the modular curve of level $3, M^{0} \simeq \mathbf{P}^{1}-\left\{1, \varepsilon, \varepsilon^{2}, \infty\right\}$. This fibration is the universal elliptic curve with a level 3 structure (once a section is chosen as origin for the group law), the 9 blown-up points corresponding to the universal points of order 3 . 
The $D(l)$ intersect in the union of 40 subvarieties $C(h)$ corresponding to the $\mathbf{Q}$ parabolics of corank 2 . The indexing set of the $h$ can be taken to be the set of lines $h \subset \mathbf{P}^{3}\left(\mathbf{F}_{3}\right)$ that are isotropic for the standard alternating form

$$
\langle x, y\rangle={ }^{t} x . J . y=\left(x_{0} y_{2}+x_{1} y_{3}\right)-\left(x_{2} y_{0}+x_{3} y_{1}\right), \quad \text { where } J=\left(\begin{array}{cc}
0 & \mathbf{1}_{2} \\
-\mathbf{1}_{2} & 0
\end{array}\right) .
$$

(A line $h$ is isotropic if $\langle x, y\rangle=0$ for all points $x, y$ on $h$.) More canonically, we take the set equivalence classes modulo \pm 1 of nonzero decomposable vectors

$$
h=x \wedge y \in \bigwedge^{2} \mathbf{F}_{3}^{4}
$$

such that the plane spanned by $x$ and $y$ in $\mathbf{F}_{3}^{4}$ is isotropic. The $C(h)$ are all disjoint from one another, and in this case each one is a union of $6 \mathbf{P}^{1}$ 's forming the 1skeleton of a tetrahedron.

Another important collection of subvarieties are the 45 Humbert surfaces $H(\Delta)$, each isomorphic with $\mathbf{P}^{1} \times \mathbf{P}^{1}$. These are the exceptional divisors that correspond to the 45 nodes on Burkhardt's quartic. The indexing set is the collection of unordered pairs $\Delta=\left\{\delta, \delta^{\perp}\right\}$, where $\delta \subset \mathbf{P}^{3}\left(\mathbf{F}_{3}\right)$ is an anisotropic line and $\delta^{\perp}$ is its dual.

Proposition 1.4. The cycle classes of the $40 \mathrm{D}(\mathrm{l})$ and the $45 H(\Delta)$ generate

$$
\mathrm{H}^{2}\left(\mathcal{A}_{2}(3)^{*}, \mathbf{Z}\right) \text {. }
$$

The 85 cycles in Prop 1.4 generate a 61-dimensional space, and the module of relations among these cycles is the unique 24-dimensional irreducible representation of $G=\mathbf{P S p}\left(4, \mathbf{F}_{3}\right)$. Indeed this is one of the 6 unipotent representations of that group. We can similarly describe generators for $\mathrm{H}^{4}\left(\mathcal{A}_{2}(3)^{*}, \mathbf{Z}\right)$. The relation of these subvarieties to classically defined loci in Burkhardt's quartic had been worked out previously by Weintraub [55]. See also [23].

1.7. To compute the cohomology of the arithmetic group $\Gamma_{2}(3)$, or in other words of the open set $\mathcal{A}_{2}(3) \subset \mathcal{A}_{2}(3)^{*}$, we use Deligne's spectral sequence

$$
\mathrm{E}_{2}^{p, q}=\mathrm{H}^{p}\left(D^{[q]}, \mathbf{Q}\right) \Rightarrow \mathrm{H}^{*}\left(\mathcal{A}_{2}(3), \mathbf{Q}\right),
$$

where $D^{[0]}=\mathcal{A}_{2}(3)^{*}$ and $D^{[q]}$ is the disjoint union of the intersections of $q$ boundary components for $q \geq 1$. The filtration defined by this spectral sequence is, up to a renumbering, the weight filtration on the mixed Hodge structure on $\mathrm{H}^{*}\left(\mathcal{A}_{2}(3), \mathbf{Q}\right)$. The sequence is degenerate in $\mathrm{E}_{3}$, and the differentials are easily computedessentially they are Gysin maps induced from the inclusions among the various intersections of boundary components. Because we have explicit generators for all the cohomologies, this comes down to understanding the intersection relations among these. We point out that Oda and Schwermer had previously analyzed this spectral sequence in this context [42].

\section{THE ZETA FUNCTION}

2.1. We compute the zeta function of Burkhardt's quartic $B$ in this section. The idea of this is the following: $B$ is birationally equivalent to a variety $C$ that admits a morphism $\pi$ to an open subset $U$ in $\mathbf{A}^{1}$ and whose typical fiber is a smooth cubic surface in $\mathbf{P}^{3}$. Understanding the zeta of $B$ reduces to that of $C$, and thus to computing the $L$-functions of the constructible sheaves $\mathrm{R}^{i} \pi_{*} \mathbf{Q}_{l}$. Since $\pi$ is smooth and proper, these are lisse, equivalent therefore to representations of $\pi_{1}(U, \bar{\eta})$. In 
other words, they are certain types of modules for $\operatorname{Gal}(\bar{\eta} / \eta)$, where $\eta=\operatorname{Spec}\left(\mathbf{F}_{q}(t)\right)$ is the generic point of $U$ and the overline denotes an algebraic closure. These are the monodromy representations on the cohomology

$$
\mathrm{H}^{i}\left(C_{\bar{\eta}}, \mathbf{Q}_{l}\right) \text {. }
$$

Since $C_{\bar{\eta}}$ is a smooth cubic surface, these cohomology groups are well-known: they have dimension 1, 7, 1 for $i=0,2,4$ and are 0 otherwise. The interesting case is $i=2$; it is generated by the cycle classes of the 27 straight lines on $C_{\bar{\eta}}$, and the Galois representation always splits as $1 \oplus \rho$ for a six dimensional $\rho$. Geometrically, this describes the effect of "analytically continuing" the 27 lines along all the paths in $U$. The key observation in our situation is that $\rho$ is trivial, which amounts to saying that all the 27 lines on $C_{\bar{\eta}}$ are in fact rational over $\operatorname{Spec}\left(\mathbf{F}_{q}(t)\right)$ when $q \equiv 1$ mod 3. This is shown by explicitly exhibiting all of them.

2.2. We let $R=\mathbf{Z}[1 / 3, \varepsilon], \varepsilon$ being a primitive third root of unity, and $K=\mathbf{Q}(\varepsilon)$ is its fraction field. In this section, we will work with schemes over $R$ unless explicitly mentioned otherwise. Let $B_{0}$ be the locus

$$
J_{4}=Y_{0}^{4}-Y_{0}\left(Y_{1}^{3}+Y_{2}^{3}+Y_{3}^{3}+Y_{4}^{3}\right)+3 Y_{1} Y_{2} Y_{3} Y_{4}=0
$$

regarded as a subscheme of $\mathbf{P}^{4}$.

Lemma 2.1. $B_{0}$ is smooth relative to $R$ in the complement of $45 R$-rational double points $b_{i}$. If $B \rightarrow B_{0}$ is the blowing-up of these points, then the exceptional fiber over each one of the $b_{i}$ is isomorphic with $\mathbf{P}^{1} \times \mathbf{P}^{1}$. The scheme $B$ is smooth above $R$.

Proof. The morphism $\varphi: B_{0} \rightarrow S=\operatorname{Spec}(\mathrm{R})$ is flat because $B_{0}$ is reduced and irreducible, $\varphi$ is dominant, and the local rings of $S$ (in maximal points) are discrete valuation rings. To check the smoothness assertions, we can verify them in the fibers $B_{0} \otimes \kappa(s)$ for all $s \in S$, and for this we use the Jacobian criterion. The derivatives are

$$
\begin{aligned}
& \frac{\partial J_{4}}{\partial Y_{0}}=4 Y_{0}^{3}-\left(Y_{1}^{3}+Y_{2}^{3}+Y_{3}^{3}+Y_{4}^{3}\right), \\
& \frac{\partial J_{4}}{\partial Y_{1}}=-3 Y_{0} Y_{1}^{2}+3 Y_{2} Y_{3} Y_{4}, \\
& \frac{\partial J_{4}}{\partial Y_{2}}=-3 Y_{0} Y_{2}^{2}+3 Y_{1} Y_{3} Y_{4}, \\
& \frac{\partial J_{4}}{\partial Y_{3}}=-3 Y_{0} Y_{3}^{2}+3 Y_{1} Y_{2} Y_{4}, \\
& \frac{\partial J_{4}}{\partial Y_{4}}=-3 Y_{0} Y_{4}^{2}+3 Y_{1} Y_{2} Y_{3} .
\end{aligned}
$$

Set these equations equal to 0 and remember that 3 is invertible. At a point $b$ in $B_{0}(\overline{\kappa(s)})$ where $Y_{0}=0$ we must have that $Y_{i}=0$ for at least 2 values of the index $i$ between 1 and 4 . If say $Y_{1}=Y_{2}=0$ then $Y_{3}^{3}+Y_{4}^{3}=0$, which gives the three points

$$
\left[0,0,0,1,-\varepsilon^{\alpha}\right], \quad \alpha=0,1,2 .
$$

Since there are 6 such choices of pairs of indices, this gives 18 singularities. (One checks also that $J_{4}=0$ at these points, a verification necessary only if char $(\kappa(s))$ 
is 2, by Euler's identity.) If $b$ is such that $Y_{0} \neq 0$, then the equations show that $Y_{i} \neq 0$ for all $i$. Then

$$
Y_{0}=\frac{Y_{2} Y_{3} Y_{4}}{Y_{1}^{2}}=\frac{Y_{1} Y_{2} Y_{3} Y_{4}}{Y_{1}^{3}}=\frac{Y_{1} Y_{2} Y_{3} Y_{4}}{Y_{2}^{3}}=\frac{Y_{1} Y_{2} Y_{3} Y_{4}}{Y_{3}^{3}}=\frac{Y_{1} Y_{2} Y_{3} Y_{4}}{Y_{4}^{3}}
$$

which gives $Y_{1}^{3}=Y_{2}^{3}=Y_{3}^{3}=Y_{4}^{3}$, and we can normalize this so that $Y_{1}=1$. This gives 27 singularities

$$
\left[\varepsilon^{\alpha+\beta+\gamma}, 1, \varepsilon^{\alpha}, \varepsilon^{\beta}, \varepsilon^{\gamma}\right], \quad \alpha, \beta, \gamma=0,1,2 .
$$

These 45 singularities clearly give disjoint sections of $\varphi$. In order to study the effect of blowing-up on these it is enough to localize around any one of them, because the finite group $G=\mathbf{P S p}\left(4, \mathbf{F}_{3}\right)$ operates transitively on the singularities (and the operations are defined over the ring $R$, cf. [4]). We therefore take $b=[1,1,1,1,1]$ and introduce affine coordinates centered there via

$$
t_{i}=Y_{i} / Y_{0}-1, \quad i=1,2,3,4
$$

The equation $J_{4}=0$ becomes

$$
\begin{aligned}
3\left(t_{1}^{2}+t_{2}^{2}+t_{3}^{2}+t_{4}^{2}-\right. & \left.\left(t_{1} t_{2}+t_{1} t_{3}+t_{1} t_{4}+t_{2} t_{3}+t_{2} t_{4}+t_{3} t_{4}\right)\right) \\
+ & \text { higher terms }=0
\end{aligned}
$$

and therefore the exceptional fiber above the point $b \in B_{0}(R)$ of the resolution $B \rightarrow B_{0}$ is isomorphic with the quadric

$$
Q=\operatorname{Proj}\left(R\left[t_{0}, t_{1}, t_{2}, t_{3}\right] / f\right),
$$

where $f=t_{1}^{2}+t_{2}^{2}+t_{3}^{2}+t_{4}^{2}-\left(t_{1} t_{2}+t_{1} t_{3}+t_{1} t_{4}+t_{2} t_{3}+t_{2} t_{4}+t_{3} t_{4}\right)$. We claim that $Q$ is isomorphic with $\mathbf{P}_{R}^{1} \times \mathbf{P}_{R}^{1}$. To see this, let

$$
\begin{aligned}
& s_{1}=t_{1}, \\
& s_{2}=t_{2}+\varepsilon t_{3}+\varepsilon^{2} t_{4}, \\
& s_{3}=-t_{1}+t_{2}+t_{3}+t_{4}, \\
& s_{4}=t_{2}+\varepsilon^{2} t_{3}+\varepsilon t_{4} .
\end{aligned}
$$

The defining equation for $Q$ becomes $s_{2} s_{4}-s_{1} s_{3}=0$. The above system of linear equations has determinant $3 \varepsilon(1-\varepsilon)$, which is invertible in the ring $R$, so that the quadric $Q$ is isomorphic over $R$ with $s_{2} s_{4}-s_{1} s_{3}=0$. This last quadric is isomorphic, even over $\mathbf{Z}$, with a product of projective lines, as can be seen from the map

$$
\left(\left[u_{0}, u_{1}\right],\left[v_{0}, v_{1}\right]\right) \mapsto\left[u_{0} v_{0}, u_{0} v_{1}, u_{1} v_{1}, u_{1} v_{0}\right] .
$$

To see that $B$ is smooth over $R$, the problem is a local one above each double point $b_{i}$, and, as before, we can take any one, for example $b=[1,1,1,1,1]$. Let $\widehat{B}$ be the formal completion of $B$ in the sheaf of ideals which is the inverse image of the defining ideal for $b$, which is $I=\left(s_{1}, s_{2}, s_{3}, s_{4}\right)$ in the coordinates introduced above. It will be enough to show that $\widehat{B}$ is smooth, because the local rings of $B$ and $\widehat{B}$ have the same completions in corresponding points of the exceptional divisor. Because blowing-up commutes with flat base extension, $\widehat{B}$ is obtained from $\operatorname{Spec}\left(R\left[\left[s_{1}, s_{2}, s_{3}, s_{4}\right]\right] / J_{4}\right)$ by blowing up the ideal $I$. However, that ring is 
isomorphic with $R\left[\left[u_{1}, u_{2}, u_{3}, u_{4}\right]\right] /\left(u_{1} u_{3}-u_{2} u_{4}\right)$ since we have $9 J_{4}=u_{1} u_{3}-u_{2} u_{4}$, where

$$
\begin{aligned}
& u_{1}=s_{1}, \\
& u_{2}=s_{2}, \\
& u_{3}=27 s_{3}+18 s_{1} s_{3}+9 s_{3}^{2}+3 s_{1}^{2} s_{3}+3 s_{1} s_{3}^{2}+s_{1}^{3}+s_{2}^{3}+s_{3}^{3}+s_{4}^{3}, \\
& u_{4}=27 s_{4}+18 s_{1} s_{4}+9 s_{3} s_{4}+3 s_{1}^{2} s_{4}+3 s_{1} s_{3} s_{4} .
\end{aligned}
$$

The Jacobian

$$
\frac{\partial\left(u_{1}, u_{2}, u_{3}, u_{4}\right)}{\partial\left(s_{1}, s_{2}, s_{3}, s_{4}\right)}
$$

is invertible in $R$ at $\left(s_{1}, s_{2}, s_{3}, s_{4}\right)=(0,0,0,0)$, so the inverse function theorem proves the isomorphism. Finally, it is clear that blowing up $u_{1} u_{3}-u_{2} u_{4}=0$ at the origin results in a smooth scheme over $R$ (just check it in the 4 natural charts).

Divide $J_{4}=0$ by $Y_{0}^{4}$, set $y_{i}=Y_{i} / Y_{0}$ for $i=1,2,3$, and let $t=Y_{4} / Y_{0}$. The defining equation becomes

$$
G=y_{1}^{3}+y_{2}^{3}+y_{3}^{3}-3 t y_{1} y_{2} y_{3}+\left(t^{3}-1\right)=0 .
$$

This defines a family of affine cubic surfaces depending on the parameter $t$. We complete this to a family of projective cubic surfaces by setting $y_{i}=x_{i} / x_{0}$ and multiplying by $x_{0}^{3}$. We get

$$
F=x_{1}^{3}+x_{2}^{3}+x_{3}^{3}-3 t x_{1} x_{2} x_{3}+\left(t^{3}-1\right) x_{0}^{3}=0 .
$$

We consider this as a closed subscheme $X$ of $\mathbf{P}_{R}^{3} \times \mathbf{A}_{R}^{1}$.

The natural projection

$$
\pi: X \longrightarrow \mathbf{A}_{R}^{1}
$$

sending ([ $\left.\left.x_{0}, x_{1}, x_{2}, x_{3}\right], t\right) \rightarrow t$ is a proper (even projective) morphism. The variety $X \otimes K$ is birationally equivalent with $B \otimes K$.

Proposition 2.2. Let $U \subset \mathbf{A}_{R}^{1}$ be the open subset where $t \neq 1, \varepsilon, \varepsilon^{2}$, i.e.

$$
U=\operatorname{Spec}\left(R\left[t,\left(t^{3}-1\right)^{-1}\right]\right) .
$$

1. $\pi$ is smooth over $U$.

2. The fibers $X_{t}$ for $t=1, \varepsilon, \varepsilon^{2}$ are each isomorphic to a union of 3 independent planes in $\mathbf{P}^{3}$.

Proof. The derivatives are

$$
\begin{aligned}
& \frac{\partial F}{\partial x_{0}}=3 x_{0}^{2}\left(t^{3}-1\right), \\
& \frac{\partial F}{\partial x_{1}}=3 x_{1}^{2}-3 t x_{2} x_{3}, \\
& \frac{\partial F}{\partial x_{2}}=3 x_{2}^{2}-3 t x_{1} x_{3}, \\
& \frac{\partial F}{\partial x_{2}}=3 x_{3}^{2}-3 t x_{1} x_{2}, \\
& \frac{\partial F}{\partial t}=-3 x_{1} x_{2} x_{3}+3 t^{2} x_{0}^{3} .
\end{aligned}
$$


(1) Let $x \in X$ be any point with $\pi(x) \in U$. We set the first four of the above equal to 0 in the residue field $\kappa(x)$. Since $3 \neq 0, t^{3} \neq 1$, we get first of all that $x_{0}=0$. We have that $t \neq 0$, because otherwise this would force $x_{1}=0, x_{2}=0, x_{3}=0$, which would not give a point in projective space. For the same reason we also must have $x_{1} x_{2} x_{3} \neq 0$, because if, say, $x_{1}=0$, it would follow from the above that $x_{2} x_{3}=0$, and these equations show that the vanishing of any two of $x_{1}, x_{2}, x_{3}$ implies the vanishing of the third. Therefore the possible singularities above $U$ can only occur where $x_{1} x_{2} x_{3} \neq 0$. But then we have

$$
x_{1}^{3}=x_{2}^{3}=x_{3}^{3}=t x_{1} x_{2} x_{3},
$$

from which we can conclude that $t$ is a cube root of unity, which is excluded. Therefore the vector of partial derivatives has maximal rank over the residue fields of all these points, which proves that these are smooth.

(2) For $t^{3}=1$ the cubic form $F$ factors into three linear factors with coefficients in $R$. For example, if $t=1$, then

$$
x_{1}^{3}+x_{2}^{3}+x_{3}^{3}-3 x_{1} x_{2} x_{3}=\left(x_{1}+x_{2}+x_{3}\right)\left(\varepsilon x_{1}+x_{2}+\varepsilon^{2} x_{3}\right)\left(\varepsilon^{2} x_{1}+x_{2}+\varepsilon x_{3}\right) .
$$

To get the decomposition for $t=\varepsilon$, merely change variables $x_{2} \rightarrow \varepsilon x_{2}$ in the above.

Let $C \subset X$ be the open subscheme where $t^{3}-1 \neq 0$. This is a family of nonsingular projective cubic surfaces parameterized by $U$.

Proposition 2.3. There are 27 closed subschemes in $C$, each isomorphic via $\pi$ with $\mathbf{P}_{U}^{1}$. In other words, the 27 straight lines on the cubic $C$ over $U$ are each rational over $U$.

Proof. In the model $G(y)=0$ they are easily found: Setting $y_{i}^{3}=1$ for $i=1,2,3$ yields a decomposable cubic form. For example, let $y_{1}=1$; then the defining equation becomes

$$
y_{2}^{3}+y_{3}^{3}-3 t y_{2} y_{3}+t^{3}=\left(\varepsilon y_{2}+y_{3}+\varepsilon^{2} t\right)\left(\varepsilon^{2} y_{2}+y_{3}+\varepsilon t\right)\left(y_{2}+y_{3}+t\right),
$$

giving 3 of the lines. For $y_{1}=\varepsilon$, for instance, one replaces $y_{2}$ by $\varepsilon y_{2}$ in the above equation. This gives 27 lines in all, and it is clear that they are distinct.

Corollary 2.4. The variety $C \otimes K$ (and hence $X \otimes K$ and $B \otimes K$ ) is a rational variety over $K$. That is, the function field is isomorphic with $K\left(w_{1}, w_{2}, w_{3}\right)$ for independent variables $w_{1}, w_{2}, w_{3}$.

Proof. One can establish rational parameters on any cubic surface $Y$ over a field $L$ in this way: Choose 2 of the 27 lines, say $N_{1}, N_{2}$, on $Y$ in such a way that they are not coplanar. This is generally possible over the algebraic closure of $L$. Define a rational map

$$
\varphi: N_{1} \times N_{2} \longrightarrow Y
$$

via

$$
\varphi(P, Q)=3 \text { rd point of intersection } R \text { of } \overline{P Q} \cap Y .
$$

That this is birational can be seen because the inverse correspondence is easily given: for any point $R$ on $Y$ not on $N_{1}$ or $N_{2}$, the plane spanned by $R$ and $N_{1}$ intersects $N_{2}$ in a unique point $Q$. The line $\overline{Q R}$ lies in the constructed plane and thus intersects $N_{1}$ in a unique point $P$. This is defined over the field $L$ provided the lines are (more precisely, provided the union of the lines is). We have precisely 
this situation with our $C \otimes K$, which we can think of as defining a cubic surface over the field $L=K(t)$ via the projection $\pi$. All 27 lines are $L$-rational by the proposition. The function field is therefore $L\left(w_{1}, w_{2}\right)$, but $L=K(t)$, so we take $w_{3}=t$.

Corollary 2.5. B, regarded as a complex projective variety, is a smooth rational threefold.

Remark 2.6. The rationality of Burkhardt's quartic was known classically (it is due to J. A. Todd). A proof can be found on 4, pp. 43-48 ]. The proof given in [54 is incorrect. (The lines meeting the planes $Y_{0}=Y_{i}=0$ for $i=1,2,3$ are contained in the hyperplane $Y_{0}=0$, and therefore so is the 4 th point of intersection-this is not the general point of the variety).

2.3. We are going to compute the zeta function of $B$ by first computing that of $C$, so we must compare these spaces. First notice that

$$
B_{0} \backslash\left\{Y_{0}=0\right\}=X \backslash\left\{x_{0}=0\right\} .
$$

The locus $Y_{0}=0$ is the union of 4 planes:

$$
\Pi_{i}=\left\{Y_{0}=Y_{i}=0\right\} \quad \text { for } i=1,2,3,4 .
$$

We take apart the locus $W=\left\{x_{0}=0\right\}$ as follows:

$$
W \subset \mathbf{P}_{R}^{2} \times \mathbf{A}_{R}^{1}
$$

is given by

$$
x_{1}^{3}+x_{2}^{3}+x_{3}^{3}-3 t x_{1} x_{2} x_{3}=0,
$$

which is the universal family of elliptic curves with a level 3 structure. We decompose this into a disjoint union: $W=W_{1} \cup W_{\infty}$, where

$$
W_{\infty}=\left\{x_{0}=x_{3}=0\right\},
$$

which is isomorphic with a disjoint union of 3 affine lines:

$$
\begin{aligned}
& M_{1}=[0,1,-1,0] \times \mathbf{A}_{R}^{1}, \\
& M_{2}=[0,1,-\varepsilon, 0] \times \mathbf{A}_{R}^{1}, \\
& M_{3}=\left[0,1,-\varepsilon^{2}, 0\right] \times \mathbf{A}_{R}^{1} .
\end{aligned}
$$

Set $\xi_{i}=x_{i} / x_{3}$ for $i=1,2$. Then $W_{1}=\left\{x_{0}=0, x_{3} \neq 0\right\} \subset \mathbf{A}_{R}^{2} \times \mathbf{A}_{R}^{1}$ is

$$
\xi_{1}^{3}+\xi_{2}^{3}+1-3 t \xi_{1} \xi_{2}=0 \text {. }
$$

This is a disjoint union $W_{1}=W_{1}^{\prime} \cup W_{1}^{\prime \prime}$, where $W_{1}^{\prime}:=\left\{\xi_{1} \xi_{2}=0\right\}$ is a union of 6 affine lines:

$$
\begin{array}{lll}
M_{4}=(0,-1) \times \mathbf{A}_{R}^{1}, & M_{5}=(0,-\varepsilon) \times \mathbf{A}_{R}^{1}, & M_{6}=\left(0,-\varepsilon^{2}\right) \times \mathbf{A}_{R}^{1}, \\
M_{7}=(-1,0) \times \mathbf{A}_{R}^{1}, & M_{8}=(-\varepsilon, 0) \times \mathbf{A}_{R}^{1}, & M_{9}=\left(-\varepsilon^{2}, 0\right) \times \mathbf{A}_{R}^{1} .
\end{array}
$$

Let $W_{1}^{\prime \prime}=\left\{\xi_{1} \xi_{2} \neq 0\right\}$. Then we have an isomorphism:

$$
\mathbf{A}_{R}^{2} \backslash\left\{\xi_{1} \xi_{2}=0\right\} \stackrel{\sim}{\longrightarrow} W_{1}^{\prime \prime}
$$

given by

$$
\left(\xi_{1}, \xi_{2}\right) \mapsto\left(\xi_{1}, \xi_{2},\left(\xi_{1}^{3}+\xi_{2}^{3}+1\right) /\left(3 \xi_{1} \xi_{2}\right)\right) .
$$


2.4. The zeta function of a scheme $V$ of finite type over the integers is

$$
\zeta_{V}(s)=\prod_{x \in|V|}\left(1-1 / N(x)^{s}\right)^{-1},
$$

where $|V|$ denotes the set of closed points of $V$ and $N(x)$ is the cardinality of the finite field $\kappa(x)=\mathcal{O}_{V, x} / \mathfrak{m}_{V, x}$. When $V$ is a scheme over the finite field $\mathbf{F}_{q}$, we can write it as

$$
\zeta_{V}(s)=Z\left(V / \mathbf{F}_{q}, q^{-s}\right)
$$

where

$$
Z\left(V / \mathbf{F}_{q}, u\right)=\exp \left(\sum_{n=1}^{\infty} \# V\left(\mathbf{F}_{q^{n}}\right) u^{n} / n\right) .
$$

It is clear that if $V$ is a disjoint union of $\mathbf{F}_{q}$ varieties $V_{1}, V_{2}$, then

$$
Z\left(V / \mathbf{F}_{q}, u\right)=Z\left(V_{1} / \mathbf{F}_{q}, u\right) Z\left(V_{2} / \mathbf{F}_{q}, u\right) .
$$

We will use Grothendieck's formula :

$$
Z\left(V / \mathbf{F}_{q}, u\right)=\prod_{i=0}^{2 d} \operatorname{det}\left(1-u F \mid \mathrm{H}_{c}^{i}\left(\bar{V}, \mathbf{Q}_{l}\right)\right)^{(-1)^{(i+1)}},
$$

where $d=\operatorname{dim} V$ and $\bar{V}=V \otimes \overline{\mathbf{F}}_{q}, \overline{\mathbf{F}}_{q}$ being an algebraic closure of $\mathbf{F}_{q} . F$ is the induced action of the Frobenius on $\mathrm{H}_{c}^{i}=$ étale cohomology with proper supports. $l$ is a prime relatively prime to $q$. In general, a bar over a variety over $\mathbf{F}_{q}$ denotes extension to the algebraic closure. Also, when clear, explicit reference to $\mathbf{F}_{q}$ will be dropped.

If $\pi: V \rightarrow U$ is an $\mathbf{F}_{q}$-morphism, we have the Leray spectral sequence with proper supports :

$$
\mathrm{E}_{2}^{a, b}=\mathrm{H}_{c}^{a}\left(\bar{U}, \mathrm{R}^{b} \pi_{!} \mathbf{Q}_{l}\right) \Rightarrow \mathrm{H}_{c}^{a+b}\left(\bar{V}, \mathbf{Q}_{l}\right)
$$

and hence

$$
Z\left(V / \mathbf{F}_{q}, u\right)=\prod_{a+b=0}^{2 d} \operatorname{det}\left(1-u F \mid \mathrm{H}_{c}^{a}\left(\bar{U}, \mathrm{R}^{b} \pi_{!} \mathbf{Q}_{l}\right)\right)^{(-1)^{(a+b+1)}} .
$$

We choose a prime power $q \equiv 1 \bmod 3$. This assures that a primitive 3 rd root of unity belongs to $\mathbf{F}_{q}$. We are going to denote by $B, C$, etc. the schemes $B \otimes \mathbf{F}_{q}, C \otimes \mathbf{F}_{q}$, etc., where $B, C$, etc. are defined in the previous section. The geometric results of the previous section hold with $R$ replaced by $\mathbf{F}_{q}$. Recall that $\pi: C \rightarrow U$ is a smooth family of cubic surfaces.

Proposition 2.7.

$$
\mathrm{R}^{i} \pi_{*} \mathbf{Q}_{l}= \begin{cases}\mathbf{Q}_{l} & \text { if } i=0 \\ \mathbf{Q}_{l}(-1)^{7} & \text { if } i=2, \\ \mathbf{Q}_{l}(-2) & \text { if } i=4, \\ 0 & \text { in all other cases. }\end{cases}
$$

Here, $(-r)$ is the Tate twist. In particular, the geometric monodromy representations are all trivial. 
Proof. Since $\pi$ is a smooth proper morphism, the sheaves $\mathrm{R}^{i} \pi_{*} \mathbf{Q}_{l}$ are lisse, and therefore equivalent to $l$-adic representations of $\pi_{1}(U, \bar{\eta})$ on

$$
\left(\mathrm{R}^{i} \pi_{*} \mathbf{Q}_{l}\right)_{\bar{\eta}}=\mathrm{H}^{i}\left(C_{\bar{\eta}}, \mathbf{Q}_{l}\right),
$$

where $\bar{\eta}=\operatorname{Spec}\left(\overline{\mathbf{F}_{q}(t)}\right)$ is a geometric generic point of $U . \pi_{1}(U, \bar{\eta})$ is the quotient of $\mathfrak{G}=\operatorname{Gal}\left(\overline{\mathbf{F}_{q}(t)} / \mathbf{F}_{q}(t)\right)$ corresponding to the maximal extension field that is unramified at all the places of $U$. Since $C_{\bar{\eta}}$ is a smooth cubic surface over an algebraically closed field, it is well known that the cohomology groups have the dimensions 1, 7, 1 for $i=0,2,4$ and 0 for all others. The only nontrivial assertion refers to the structure for $i=2$. Recall that $\mathrm{H}^{2}\left(C_{\bar{\eta}}, \mathbf{Q}_{l}\right)$ is generated by the cycle classes of the lines on the cubic. In our case, these lines are $L=\mathbf{F}_{q}(t)$-rational by proposition 2.3. so that it is clear that the geometric monodromy is trivial (analytic continuation of any line along a loop in $U$ brings the line back to itself). More precisely, the action of $\pi_{1}(U, \bar{\eta})$ is just the induced action of $\mathfrak{G}=\operatorname{Gal}(\bar{L} / L)$. The result we need follows from

Lemma 2.8. Let $V_{0}$ be a smooth proper variety over a field L. Suppose that

$$
\mathrm{H}^{2 i}\left(V_{0} \otimes \bar{L}, \mathbf{Q}_{l}\right)
$$

is generated by the classes of codimension $i$ algebraic cycles rational over $L$. Then, as a $\mathfrak{G}$-module, $\mathrm{H}^{2 i}\left(V_{0} \otimes \bar{L}, \mathbf{Q}_{l}\right)$ is isomorphic with a direct sum of copies of $\mathbf{Q}_{l}(-i)$.

Proof. This is also well known. The point is that the cycle class map

$$
\mathfrak{Z}^{i}\left(V_{0}\right) \longrightarrow \mathrm{H}^{2 i}\left(V_{0} \otimes \bar{L}, \mathbf{Q}_{l}\right)(i)
$$

is $\mathfrak{G}$-equivariant, and hence the image lands in the $\mathfrak{G}$-invariants. This proves the claim.

This completes the proof of the proposition.

Remark 2.9. 1. The lines on a cubic surface $V$ generate

$$
\operatorname{Pic}(\mathrm{V}) \simeq \mathbf{Z}^{7} .
$$

This can be seen by considering a "double six"

$$
e_{1}, e_{2}, e_{3}, e_{4}, e_{5}, e_{6}, g_{1}, g_{2}, g_{3}, g_{4}, g_{5}, g_{6}
$$

and considering the intersection matrix of $g_{1}, e_{1}, e_{2}, e_{3}, e_{4}, e_{5}, e_{6}$, which has determinant 4 (see the discussion in [19, pp. 401-406]). This shows that the lines generate a subgroup of index at most 2 . It is known that $l, e_{1}, e_{2}, e_{3}, e_{4}, e_{5}, e_{6}$ generate the Picard group, where $l$ is the class defined in [19, p. 401], and that the class $h$ of a hyperplane is $3 l-\sum e_{i}$. But it is also known that there are hyperplane sections consisting of three lines (in fact there are 45 such "tritangent planes"), so that $h$ belongs to the subgroup generated by the lines. It follows that $3 l$ belongs to that subgroup, and because the index is at most 2 , in fact $l$ itself is in there.

2. From the point of view of the theory of motives, the above result can be understood as follows: If $C$ is a cubic surface over a field $L$, then its motive has a decomposition into its parts of pure weight $h^{i}(C)$ for $i=0,2,4$. One knows that

$$
h^{2}(C)=(1 \oplus \rho) \otimes \mathbf{Q}(-1),
$$

where 1 is generated by the hyperplane class, and $\rho$ is an Artin motive coming from the action of $\operatorname{Gal}(\bar{L} / L)$ on the 27 lines on $C \otimes \bar{L}$. (see Serre's article in [49]). In our case, $\rho=1^{6}$. 


\section{Corollary 2.10.}

$$
Z\left(C / \mathbf{F}_{q}, u\right)=\frac{(1-u)^{3}(1-q u)^{20}}{\left(1-q^{2} u\right)^{4}\left(1-q^{3} u\right)} .
$$

Proof. By Grothendieck's formula $\left(\pi_{!}=\pi_{*}\right.$, since $\pi$ is a proper morphism) and the above proposition, it all comes down to computing

$$
\operatorname{det}\left(1-u F \mid \mathrm{H}_{c}^{a}\left(\bar{U}, \mathbf{Q}_{l}(-r)\right)\right)=\operatorname{det}\left(1-q^{r} u F \mid \mathrm{H}_{c}^{a}\left(\bar{U}, \mathbf{Q}_{l}\right)\right) .
$$

(The effect of the Tate twist $(-r)$ is to replace $u$ by $q^{r} u$.) These are known:

$$
\operatorname{det}\left(1-u F \mid \mathrm{H}_{c}^{a}\left(\bar{U}, \mathbf{Q}_{l}\right)\right) \text { is } 0,(1-u)^{3},(1-q u) \text { for } a=0,1,2
$$

This can easily be seen by simply counting the rational points on $U$, applying Grothendieck's formula, and using the known facts that

$$
\mathrm{H}_{c}^{0}\left(\bar{U}, \mathbf{Q}_{l}\right)=0 \quad \text { and } \quad \mathrm{H}_{c}^{2}\left(\bar{U}, \mathbf{Q}_{l}\right)=\mathbf{Q}_{l}(-1)
$$

[12, p. 282].

$C$ is obtained from $X$ by removing the singular fibers $X_{t}$ for $t=1, \varepsilon, \varepsilon^{2}$, and each of these is a union of 3 planes in $\mathbf{P}^{3}$. The zeta functions of the latter are easily computed:

$$
Z\left(X_{t}, u\right)=\frac{1}{(1-u)\left(1-q^{2} u\right)^{3}} .
$$

Therefore

$$
Z(X, u)=Z(C, u) \prod_{t=1, \varepsilon, \varepsilon^{2}} Z\left(X_{t}, u\right)=\frac{(1-q u)^{20}}{\left(1-q^{2} u\right)^{13}\left(1-q^{3} u\right)} .
$$

We therefore get

$$
Z\left(B_{0}, u\right)=Z(X, u) Z(W, u)^{-1} Z\left(Y_{0}=0, u\right)
$$

and

$$
\begin{gathered}
Z(W, u)=Z\left(W_{\infty}, u\right) Z\left(W_{1}^{\prime}, u\right) Z\left(W_{1}^{\prime \prime}, u\right), \\
Z\left(Y_{0}=0, u\right)=\prod_{i=0}^{3} Z\left(\Pi_{i}, u\right) \prod_{i<j} Z\left(\Pi_{i} \cap \Pi_{j}, u\right)^{-1} \\
\times \prod_{i<j<k} Z\left(\Pi_{i} \cap \Pi_{j} \cap \Pi_{k}, u\right) .
\end{gathered}
$$

There are 4 planes $\Pi_{i}, 6$ lines $\Pi_{i} \cap \Pi_{j}, 4$ points $\Pi_{i} \cap \Pi_{j} \cap \Pi_{k}$, and $\Pi_{1} \cap \Pi_{2} \cap \Pi_{3} \cap \Pi_{4}$ is empty. Hence

$$
Z\left(Y_{0}=0, u\right)=\frac{(1-q u)^{2}}{(1-u)^{2}\left(1-q^{2} u\right)^{4}}
$$

We have:

$$
\begin{aligned}
Z\left(W_{\infty}, u\right) & =\prod_{i=1}^{3} Z\left(M_{i}, u\right), \\
Z\left(W_{1}^{\prime}, u\right) & =\prod_{i=4}^{9} Z\left(M_{i}, u\right) .
\end{aligned}
$$


Each $M_{i}$ is an affine line, so

$$
Z\left(M_{i}, u\right)=\frac{1}{1-q u}
$$

and

$$
Z\left(W_{1}^{\prime \prime}, u\right)=Z\left(\mathbf{A}^{2} \backslash \text { coordinate axes, } u\right)=\frac{(1-q u)^{2}}{(1-u)\left(1-q^{2} u\right)} .
$$

Putting it all together gives

$$
Z\left(B_{0}, u\right)=\frac{(1-q u)^{29}}{(1-u)\left(1-q^{2} u\right)^{16}\left(1-q^{3} u\right)} .
$$

$B$ is obtained by resolving the 45 nodes on $B_{0}$. The effect of blowing up any one of these is to replace one $\mathbf{F}_{q}$-rational point by a $\mathbf{P}^{1} \times \mathbf{P}^{1}$. Each singularity contributes a factor

$$
\begin{aligned}
Z\left(\mathbf{P}^{1} \times \mathbf{P}^{1}, u\right) Z(\text { Point }, u)^{-1} & =\frac{1}{(1-u)(1-q u)^{2}\left(1-q^{2} u\right)}(1-u) \\
& =\frac{1}{(1-q u)^{2}\left(1-q^{2} u\right)} .
\end{aligned}
$$

Therefore

$$
Z(B, u)=Z\left(B_{0}, u\right)\left(\frac{1}{(1-q u)^{2}\left(1-q^{2} u\right)}\right)^{45}
$$

This proves

Theorem 2.11. Let $B$ be the projective and smooth variety obtained by resolving the 45 nodes of the projective hypersurface with equation

$$
Y_{0}^{4}-Y_{0}\left(Y_{1}^{3}+Y_{2}^{3}+Y_{3}^{3}+Y_{4}^{3}\right)+3 Y_{1} Y_{2} Y_{3} Y_{4}=0 .
$$

Let $q$ be a prime power congruent to 1 modulo 3. Then

$$
Z\left(B / \mathbf{F}_{q}, u\right)=\frac{1}{(1-u)(1-q u)^{61}\left(1-q^{2} u\right)^{61}\left(1-q^{3} u\right)} .
$$

Corollary 2.12. Let $A_{2}(3)^{*}$ denote the underlying complex manifold of $\mathbf{C}$-valued points of the Igusa compactification of the moduli space of principally polarized abelian varieties of dimension 2 with a level 3 structure. Then the dimensions of

$$
\mathrm{H}^{i}\left(A_{2}(3)^{*}, \mathbf{Q}\right)
$$

are 1, 0,61, 0,61, 0, 1 for $i=0, \ldots, 6$, and 0 for all other values .

Proof. Since $B \otimes \mathbf{C}$ is isomorphic with $A_{2}(3)^{*}$, the result follows from the comparison theorem in étale cohomology.

The form of the zeta function suggests that the cohomology is generated by algebraic cycles. We will see that this is indeed the case.

Corollary 2.13. Regard $B$ as a projective smooth variety over the number field $K=\mathbf{Q}(\varepsilon)$. Then the Hasse-Weil zeta function of $B$ is

$$
\zeta_{K}(s) \zeta_{K}(s-1)^{61} \zeta_{K}(s-2)^{61} \zeta_{K}(s-3),
$$

where $\zeta_{K}(s)$ is the Dedekind zeta function of the field $K$. 
Proof. The only unproven assertion concerns the form of the Euler factors at the unique prime of $K$ above 3 . According to Serre [48] these are given by the action of the corresponding Frobenius on the $I_{3}$-invariants in the $l$-adic cohomology groups, where $I_{3} \subset \operatorname{Gal}(\overline{\mathrm{K}} / \mathrm{K})$ is the inertia subgroup at this place (well-defined up to conjugacy, $l \neq 3)$. But the results proven show that these representations are sums of twists of the canonical action on the $l^{n}$ th roots of unity, and the 3 -inertia is trivial on this.

Remark 2.14. The above calculations show that the zeta function of the affine variety

$$
y_{1}^{3}+y_{2}^{3}+y_{3}^{3}-3 t y_{1} y_{2} y_{3}+\left(t^{3}-1\right)=0
$$

is

$$
Z\left(B_{0} \backslash\left\{Y_{0}=0\right\}, u\right)=\frac{(1-u)(1-q u)^{27}}{\left(1-q^{2} u\right)^{12}\left(1-q^{3} u\right)} .
$$

This predicts that the number of solutions to the above equation in the finite field $\mathbf{F}_{q}$ is $q^{3}+12 q^{2}-27 q-1$. This has been checked by a computer search for the primes $q=7,13$ and 19, giving respectively 741, 3873 and 10677 points.

\section{CyCles}

Important algebraic subvarieties of Siegel modular threefolds are indexed by a combinatorial structure called the Tits building with scaffolding. We will describe this finite geometry and the cycles parameterized by it in this section. We will state the results for an arbitrary level $n \geq 3$. We consider the module $(\mathbf{Z} / n)^{4}$ with the standard alternating form $\langle$,$\rangle (see the introduction). A submodule M \subset(\mathbf{Z} / n)^{4}$ is isotropic if $\langle x, y\rangle=0$ for all $x, y \in M$. Consider the following finite sets:

$$
\begin{aligned}
& \mathfrak{P}_{1}(\mathbf{Z} / n)=\left\{\begin{array}{l}
\text { nonzero vectors } l \in(\mathbf{Z} / n)^{4} \text { modulo } \pm 1 \\
\text { such that the submodule generated by } l \\
\text { is a free direct factor }
\end{array}\right\}, \\
& \mathfrak{P}_{2}(\mathbf{Z} / n)=\left\{\begin{array}{l}
\text { nonzero decomposable vectors } \\
h=v_{1} \wedge v_{2} \in \bigwedge^{2}(\mathbf{Z} / n)^{4} \\
\text { modulo } \pm 1, \text { such that the submodule } \\
\tilde{h} \text { generated by } v_{1}, v_{2} \\
\text { is an isotropic free direct factor }
\end{array}\right\}, \\
& \mathfrak{P}_{1,2}(\mathbf{Z} / n)=\left\{\begin{array}{l}
\text { pairs }(l, h), \text { with } l, h \text { as above, such that } \\
l \text { is a direct factor of } \tilde{h}
\end{array}\right\}, \\
& \mathfrak{Q}(\mathbf{Z} / n)=\left\{\begin{array}{l}
\text { unordered pairs } \Delta=\left\{\delta, \delta^{\perp}\right\}, \\
\text { where } \delta \subset(\mathbf{Z} / n)^{4} \text { is a free summand, } \\
\text { on which }\langle,\rangle \text { is nonsingular } \\
\text { and } \delta^{\perp} \text { is its orthogonal complement }
\end{array}\right\} .
\end{aligned}
$$

A vector $l=\left(l_{1}, l_{2}, l_{3}, l_{4}\right)$ will generate a free direct factor precisely when $\left(l_{1}, l_{2}, l_{3}, l_{4}\right)$ is the unit ideal in $\mathbf{Z} / n$. The submodule $\tilde{h}$ will be a free direct factor precisely when the six Plücker coordinates of $h$ generate the unit ideal in $\mathbf{Z} / n$. Note that when $n$ has two or more prime factors, there are direct factors of $\mathbf{Z} / n$ that are not free. The elements $\Delta$ are called nonsingular pairs. Each provides a splitting

$$
(\mathbf{Z} / n)^{4}=\delta \oplus \delta^{\perp}
$$


into free submodules of rank 2 mutually dual relative to the alternating form. In the cases $n=3$ and $n=4$, the units of the $\operatorname{ring} \mathbf{Z} / n$ consist only of \pm 1 , and in these cases, we may identify the above objects with subsets of the projective space $\mathbf{P}^{3}(\mathbf{Z} / n)$. Indeed,

$$
\mathbf{P}^{3}(\mathbf{Z} / n)=\mathfrak{P}_{1}(\mathbf{Z} / n)
$$

for $n=3,4$, and the elements of $\mathfrak{P}_{2}(\mathbf{Z} / n)$ (resp. $\mathfrak{Q}(\mathbf{Z} / n)$ ) are certain kinds of lines (resp. pairs of lines) in that projective 3 -space.

These sets have the cardinalities

$$
\begin{aligned}
\# \mathfrak{P}_{1}(\mathbf{Z} / n) & =\left(n^{4} / 2\right) \prod_{p \mid n}\left(1-p^{-4}\right) \\
\# \mathfrak{P}_{2}(\mathbf{Z} / n) & =\left(n^{4} / 2\right) \prod_{p \mid n}\left(1-p^{-4}\right) \\
\# \mathfrak{P}_{1,2}(\mathbf{Z} / n) & =\left(n^{6} / 4\right) \prod_{p \mid n}\left(1-p^{-2}\right)\left(1-p^{-4}\right), \\
\# \mathfrak{Q}(\mathbf{Z} / n) & =\left(n^{4} / 2\right) \prod_{p \mid n}\left(1+p^{-2}\right) .
\end{aligned}
$$

There is an obvious notion of incidence among the configurations introduced here, which coincides with the usual notion of incidence in projective space when $n=3,4$. The number of $l$ 's on each $h$ is the same as the number of $h$ 's on each $l$, which is

$$
\left(n^{2} / 2\right) \prod_{p \mid n}\left(1-p^{-2}\right) .
$$

The sets and their incidence relations form the Tits building with scaffolding.

The intrinsic definition of the sets $\mathfrak{P}(\mathbf{Z} / n)$ is that they index the $\Gamma_{2}(n)$-equivalence classes of nontrivial Q-parabolic subgroups of $\mathbf{S p}(4)$. Recall that apart from $\mathbf{S p}(4)$ itself there are three classes of such - two maximal ones, and the Borel subgroups. The intrinsic definition of $\mathfrak{Q}(\mathbf{Z} / n)$ is the set of $\Gamma_{2}(n)$-equivalence classes of certain kinds of involutions in $\mathbf{S p}(4, \mathbf{Q})$ (see [26]).

Finally we note that the group $\mathbf{S p}(4, \mathbf{Z} / n)$ acts transitively on each of the four sets here.

3.1. The boundary. The boundary of the Igusa compactification is a divisor with normal crossings:

$$
\partial \mathcal{A}_{2}(n)^{*}=\mathcal{A}_{2}(n)^{*}-\mathcal{A}_{2}(n)=\bigcup_{l} D(l), \quad l \in \mathfrak{P}_{1}(\mathbf{Z} / n) .
$$

Each boundary component $D(l)$ is an elliptic modular surface $D$ of level $n$. These surfaces have been studied by Shioda [51]. $D$ has the following structure: Let $M^{0}=$ $\Gamma_{1}(n) \backslash \mathfrak{S}_{1}$ be the modular curve of level $n$, and let $M$ be the smooth compactification of it. The genus is given by $g(M)=1+(n-6) \mu(n) / 12 n$, where

$$
\mu(n)=\#\left(\mathbf{S L}_{2}(\mathbf{Z} / n) / \pm 1\right)=\left(n^{3} / 2\right) \prod_{p \mid n}\left(1-p^{-2}\right)
$$

The complement $M \backslash M^{0}$ consists of $t(n)=\mu(n) / n$ cusps. There is a natural morphism $\pi: D \rightarrow M$ which is a compactification of the universal elliptic curve with a level $n$ structure $D^{0} \rightarrow M^{0}(n \geq 3)$. $\pi$ has $n^{2}$ sections corresponding to the points of order $n$ on the universal elliptic curve. Over each cusp $s$ the fiber $\pi^{-1}(s)$ 
is an $n$-gon of $\mathbf{P}^{1}$ 's. In Kodaira's classification, this is of type $I_{n}$. The following theorem gives the topology of $D$ :

Theorem 3.1 ([51]). 1. $\pi^{*}$ induces an isomorphism $\mathrm{H}^{1}(M, \mathbf{Q}) \simeq \mathrm{H}^{1}(D, \mathbf{Q})$.

2. $\mathrm{H}^{2,0}(D)=\mathrm{H}^{0}\left(D, \Omega_{D}^{2}\right)$ is naturally isomorphic with the space of cusp forms of weight 3 for $\Gamma_{1}(n)$. Hence, $h^{2,0}=h^{0,2}=(n-3) \mu(n) / 6 n$.

3. $\mathrm{H}^{1,1}(D)=\mathrm{NS}(\mathrm{D}) \otimes \mathbf{C}$. Therefore $\rho=\mathrm{h}^{1,1}=2+(n-1) \mu(n) / n$.

4. The Néron-Severi group is generated by the $\mathbf{P}^{1}$ 's lying over the cusps and the $n^{2}$ sections.

The first part gives a natural identification of $\mathrm{H}^{1,0}(D)$ with the space of cusp forms of weight 2 for $\Gamma_{1}(n)$. In fact, $D$ and $M$ have canonical models over the ring $\mathbf{Z}[1 / n, \varepsilon]$, where $\varepsilon$ is a primitive $n^{\text {th }}$ root of unity. For each prime $\mathfrak{p}$ of this ring we can consider the zeta function of $D$ over the finite field $\mathbf{F}_{q}$ with $q=\mathrm{Np}$. This can be given explicitly in terms of the Hecke polynomials $\operatorname{det}\left(1-T_{p} u+p^{w-1} R_{p} u^{2}\right)$ acting on the spaces of cusp forms of weight $w=2,3$ for $\Gamma_{1}(n)$. The precise form of it is given in Shioda's paper. Actually, to get a simple formula one might have to enlarge the field to $\mathbf{F}_{q^{2}}$ because in general the $\mathbf{P}^{1}$ 's lying over the cusps are only defined over that field.

One can give a set of generators for the Néron-Severi group $\otimes \mathbf{Q}$ as follows: Choose any one of the $n^{2}$ sections. Then over each cusp take the $n-1$ of the $n \mathbf{P}^{1}$ 's not meeting this section. Finally adjoin any general fiber of $\pi$. This set of generators is somewhat asymmetrical. One can write down all the intersection relations of these various cycles, but to do so requires a good indexing system. One such is this: First observe that the cusps of the modular curve $M$ are indexed by the primitive pairs $(a, b) \in(\mathbf{Z} / n)^{2}$ (primitive means that $a$ and $b$ generate the unit ideal in $\mathbf{Z} / n$, or equivalently the vector $(a, b)$ generates a direct factor). These are identified if the vectors differ by multiplication by \pm 1 . We can index the $\mathbf{P}^{1}$ 's as $E(a, b, c)$ for a vector $(a, b, c) \in(\mathbf{Z} / n)^{3}$ such that $(b, c)$ is primitive, and these are also identified modulo multiplication by \pm 1 . $E(a, b, c)$ projects to the cusp $(b, c)$. The intersections of these are given by

$$
E\left(a_{1}, b_{1}, c_{1}\right) \cdot E\left(a_{2}, b_{2}, c_{2}\right)= \begin{cases}1 & \text { if }\left(a_{2}, b_{2}, c_{2}\right) \equiv \pm\left(a_{1} \pm 1, b_{1}, c_{1}\right) \bmod n \\ -2 & \text { if }\left(a_{2}, b_{2}, c_{2}\right) \equiv \pm\left(a_{1}, b_{1}, c_{1}\right) \bmod n \\ 0 & \text { otherwise }\end{cases}
$$

The $n^{2}$ sections are indexed by vectors $(\alpha, \beta) \in(\mathbf{Z} / n)^{2}$, or, better, by the linear forms $X+\alpha Y+\beta Z$. Then

$$
S(\alpha, \beta) \cdot E(a, b, c)= \begin{cases}1 & \text { if } a+\alpha b+\beta c \equiv 0 \bmod n, \\ 0 & \text { otherwise. }\end{cases}
$$

Since the sections are disjoint from each other, to complete our description of the intersection relations we need to give the self-intersection of a section. This is:

Proposition 3.2. $S(\alpha, \beta)^{2}=-\chi\left(\mathcal{O}_{D}\right)=-\mu(n) / 12$.

Proof. Let $S$ be a section, regarded as a divisor on $D$, and let $\mathcal{L}=\mathcal{O}_{D}(S)$. By the Riemann-Roch theorem,

$$
\chi(D, \mathcal{L})=S .\left(S-K_{D}\right) / 2+\chi\left(O_{D}\right)
$$


where $K_{D}$ is the canonical divisor on $D$. According to Shioda [51],

$$
K_{D}=\pi^{*}\left(\mathcal{O}_{M}(\mathfrak{k}-\mathfrak{f})\right),
$$

where $\mathfrak{k}$ is the canonical class on the modular curve $M$ and $\mathcal{O}_{M}(\mathfrak{f})$ is the sheaf on $M$ which is the normal bundle of the imbedding of $S \simeq M$ into $D$. It follows that the degree of the divisor $\mathfrak{f}$ is $S^{2}$. Therefore, $S .\left(S-K_{D}\right)=S^{2}-\operatorname{deg}(\mathfrak{k})+\operatorname{deg}(\mathfrak{f})=$ $2 S^{2}-2 g(M)+2$. The Leray spectral sequence gives

$$
\chi(D, \mathcal{L})=\chi\left(M, \mathbf{R} \pi_{*} \mathcal{L}\right) .
$$

We have $\pi_{*} \mathcal{L}=\mathcal{O}_{M}$ and $\mathrm{R}^{i} \pi_{*} \mathcal{L}=0$ for $i>0$. This can be seen fiber-by-fiber, where it amounts to the well-known assertion that on an elliptic curve $E$ or an $n$-gon of $\mathbf{P}^{1}$ s, $\mathrm{H}^{i}(E, \mathcal{O}(P))$ is 0 for $i>0$ and consists of constants for $i=0$. The assertion then follows from standard base-changing theorems. This gives us

$$
\chi(D, \mathcal{L})=\chi\left(M, \mathcal{O}_{M}\right)=1-g(M) .
$$

The claimed result then follows by comparing both sides in the Riemann-Roch formula.

The boundary components intersect in the disjoint union of spaces $C(h)$ corresponding to the $h \in \mathfrak{P}_{2}(\mathbf{Z} / n)$. Each $C(h)$ is a union of projective lines. Replacing each $\mathbf{P}^{1}$ by a line segment $[0,1]$ yields a space which is the 1 -skeleton for a tessellation of the Riemann surface $\mathcal{A}_{1}(n)^{*}$ into $n$-gons. Fixing $h$, the faces of this tessellation correspond to the l's that are on $h$ (i.e. such that the submodule generated by $l$ is a direct factor of $\tilde{h}$ ), the edges correspond to the unordered pairs $\left(l_{1}, l_{2}\right)$ such that $l_{1} \wedge l_{2}=h$, and the vertices correspond to the unordered triples $\left(l_{1}, l_{2}, l_{3}\right)$ such that $l_{1} \wedge l_{2}=l_{1} \wedge l_{3}=l_{2} \wedge l_{3}=h$ (recall that these are identified modulo \pm 1 ). We see that there are respectively

$$
\left(n^{2} / 2\right) \prod_{p \mid n}\left(1-p^{-2}\right), \quad\left(n^{3} / 4\right) \prod_{p \mid n}\left(1-p^{-2}\right), \quad\left(n^{3} / 6\right) \prod_{p \mid n}\left(1-p^{-2}\right)
$$

faces, edges and vertices, showing that the Riemann surface has exactly the same genus as that of the modular curve $M$ of level $n$. This can be more clearly seen by considering the dual cell decomposition; the vertices then actually correspond to the cusps of the modular curve.

One can view the boundary $\partial \mathcal{A}_{2}(3)^{*}$ this way: Start with a disjoint union of the $40 C(h)$, each of which is a tetrahedron of projective lines, and think of this as a collection of sockets. Plug the $40 D(l)$ into these sockets in such a way that a cuspidal triangle of projective lines fills in a face of one of the $C(h)$. Of course, the global arrangement of these is governed by the incidences in the finite geometry $\mathfrak{P}(\mathbf{Z} / n)$.

3.2. The Humbert surfaces. $\mathfrak{Q}(\mathbf{Z} / n)$ indexes the Humbert surfaces on $\mathcal{A}_{2}(n)^{*}$. These are the fixed points of certain kinds of involutions in the integral symplectic group. It is known that each is isomorphic with the product of the modular curve of that level with itself:

$$
\left(\Gamma_{1}(n) \backslash \mathfrak{S}_{1}\right)^{*} \times\left(\Gamma_{1}(n) \backslash \mathfrak{S}_{1}\right)^{*}=\mathcal{A}_{1}(n)^{*} \times \mathcal{A}_{1}(n)^{*} .
$$

Summarizing, we get 
Proposition 3.3. The Humbert surfaces $H(\Delta) \subset \mathcal{A}_{2}(n)^{*}$ are indexed by the nonsingular pairs $\Delta=\left\{\delta, \delta^{\perp}\right\}$, where $\delta \subset(\mathbf{Z} / n)^{4}$ is a free anisotropic summand of rank 2. This indexing is equivariant for the natural action of $\mathbf{S p}_{4}(\mathbf{Z} / n)$ on both $\mathcal{A}_{2}(n)^{*}$ and $(\mathbf{Z} / n)^{4}$ :

$$
g \cdot H(\Delta)=H(g . \Delta) .
$$

Each $H(\Delta)$ is isomorphic with $\mathcal{A}_{1}(n)^{*} \times \mathcal{A}_{1}(n)^{*}$, and they are all disjoint from one another. There are

$$
\left(n^{4} / 2\right) \prod_{p \mid n}\left(1+p^{-2}\right)
$$

Humbert surfaces.

Remark 3.4. The Humbert surfaces inside the moduli space $\mathcal{A}_{2}(n)$ have the following interpretation: they are the loci along which the principally polarized abelian surfaces with their level $n$ structures split into products of elliptic curves. Humbert surfaces are more generally defined; roughly these correspond to loci where the corresponding abelian surface acquires nontrivial endomorphisms. For each positive integer $d$ there are Humbert surfaces of discriminant $d$, and we are considering only those where $d=1$. Those where $d$ is not a square are Hilbert modular surfaces imbedded in the Siegel modular threefold. For a study of these, see [17].

We give the intersections of the Humbert surfaces with the boundary components. In what follows, notation such as $l \in \delta$ means that the vector $l$ generates a free summand of $\delta$.

Proposition 3.5. $H(\Delta) \cap D(l)=\emptyset$ unless $l \in \delta$ or $l \in \delta^{\perp}$ as a free summand, where $\Delta=\left\{\delta, \delta^{\perp}\right\}$. If nonempty, it is transverse and of the form

$$
\text { cusp } \times \mathcal{A}_{1}(n)^{*} \text { or } \mathcal{A}_{1}(n)^{*} \times \text { cusp }
$$

on $H(\Delta)=\mathcal{A}_{1}(n)^{*} \times \mathcal{A}_{1}(n)^{*}$, and it is one of the $n^{2}$ sections on the elliptic modular surface $D(l)$. Every divisor on $H(\Delta)$ of the form cusp $\times \mathcal{A}_{1}(n)^{*}$ or $\mathcal{A}_{1}(n)^{*} \times$ cusp is an intersection with a boundary component, and every one of the $n^{2}$ sections on $D(l)$ in an intersection with a Humbert surface.

From the point of view of the combinatorics of the Tits building these last conditions can be expressed as follows: There are $t(n)=\mu(n) / n$ primitive $\pm l$ on (i.e., generating a free summand of) each anisotropic free summand $\delta \subset(\mathbf{Z} / n)^{4}$ of rank 2 (indeed, on any free summand of rank 2), and there are $n^{2}$ anisotropic free summands $\delta \subset(\mathbf{Z} / n)^{4}$ containing any primitive $\pm l$ as a free direct factor.

When $n$ is 3 , the $l, h, \delta$ can be identified with configurations in $\mathbf{P}^{3}(\mathbf{Z} / 3)$, and one can visualize the incidences in terms of the finite projective space in this way: The $\pm l$ are the 40 points in that projective 3 -space. The set of points $m$ such that $\langle m, l\rangle=0$ is a hyperplane $\Pi_{l} \simeq \mathbf{P}^{2}(\mathbf{Z} / 3)$ containing $l$. The 12 different $\mathbf{P}^{1}$ 's on $D(l)$ lying over cusps correspond to the $m$ 's on $\Pi_{l}$ other than $l$. The isotropic lines $h$ containing $l$ are the lines through $l$ in $\Pi_{l}$, and these correspond to the triangles of $\mathbf{P}^{1}$ lying over the cusps. The $9 \delta$ 's incident upon $l$, corresponding to the 9 sections of the surface $D(l)$, are the 9 lines through $l$ in $\mathbf{P}^{3}(\mathbf{Z} / 3)$ not contained in $\Pi_{l}$. Their duals $\delta^{\perp}$ are the 9 lines in $\Pi_{l}$ not passing through $l$.

One should be aware that, for a general $n$, the map from the $\pm l$ to the points of projective space is many-to-one (it is $\varphi(n) / 2$-to-one with Euler's $\varphi$ ), so that the incidence geometry cannot be directly visualized inside a projective space over $\mathbf{Z} / n$. 
These intersection properties explain the indexing of the divisors on an elliptic modular surface that was used in the previous section. Thinking of this surface as the boundary component $D(1,0,0,0)$, then the projective lines lying over the cusps are the primitive $\pm l$ such that $(1,0,0,0) \wedge l$ gives a free isotropic direct summand in $V(\mathbf{Z} / n)$. This comes down to $l=(a, b, 0, c)$ with $(b, c)$ primitive. Two of these will intersect transversally iff $l_{1} \wedge l_{2} \equiv \pm(1,0,0,0) \wedge l_{1} \equiv \pm(1,0,0,0) \wedge l_{2} \bmod n$, which implies that $\left(a_{2}, b_{2}, c_{2}\right) \equiv \pm\left(a_{1} \pm 1, b_{1}, c_{1}\right) \bmod n$. The self-intersection of these divisors being -2 is a known property of the Kodaira classification. In the same way, the $n^{2}$ sections are identified with the $\Delta$ 's incident upon $(1,0,0,0)$, and these can be thought of as the lines in the plane

$$
\Pi_{(1,0,0,0)}=\{(x, y, 0, z)\}
$$

"not passing through $(1,0,0,0)$ ", that is, forming a free complement to $(1,0,0,0)$. These can be identified with the lines $X+\alpha Y+\beta Z=0$.

\subsection{Intersection numbers. We define}

$$
D\left(l_{1}, l_{2}\right)=D\left(l_{1}\right) \cap D\left(l_{2}\right) \text { for } l_{1} \wedge l_{2} \in \mathfrak{P}_{2}(\mathbf{Z} / n) \text {. }
$$

These are projective lines, and there are $\left(n^{7} / 8\right) \prod_{p \mid n}\left(1-p^{-2}\right)\left(1-p^{-4}\right)$ of them. In a previous notation,

$$
D((1,0,0,0),(a, b, 0, c))=E(a, b, c)
$$

We set

$$
D\left(l_{1}, l_{2}, l_{3}\right)=D\left(l_{1}\right) \cap D\left(l_{2}\right) \cap D\left(l_{3}\right) \text { for } l_{1} \wedge l_{2}=l_{1} \wedge l_{3}=l_{2} \wedge l_{3} \in \mathfrak{P}_{2}(\mathbf{Z} / n) .
$$

These are points, and there are $\left(n^{7} / 12\right) \prod_{p \mid n}\left(1-p^{-2}\right)\left(1-p^{-4}\right)$ of them. No more than 3 boundary components can have an intersection. We set

$$
S(l, \Delta)=D(l) \cap H(\Delta) \quad \text { whenever } l \in \Delta \text {, i.e. } l \in \delta \text { or } l \in \delta^{\perp}
$$

as free summands (in a notation such as $l \in \delta$, it is always understood that $l$ is a free direct summand of $\delta$ ). These are isomorphic with $M=\mathcal{A}_{1}(n)^{*}$. There are $\left(n^{6} / 2\right) \prod_{p \mid n}\left(1-p^{-4}\right)$ of them. We set

$$
F(l)=\text { a general fiber of } D(l) \rightarrow B(l) .
$$

These are genus 1 curves, and there are $\left(n^{4} / 2\right) \prod_{p \mid n}\left(1-p^{-4}\right)$ of them.

We determine the intersection numbers of the divisors $D(l), H(\Delta)$ with the codimension 2 subvarieties $D\left(l_{1}, l_{2}\right), S(l, \Delta), F(l)$.

Lemma 3.6.

$$
D(l) \cdot D\left(l_{1}, l_{2}\right)= \begin{cases}1 & \text { if } l \wedge l_{1}=l \wedge l_{2}=l_{1} \wedge l_{2} \in \mathfrak{P}_{2}(\mathbf{Z} / n), \\ -2 & \text { if } l=l_{1} \text { or } l_{2} \\ 0 & \text { otherwise. }\end{cases}
$$

Proof. The first assertion is a restatement of the fact that 3 boundary components will meet in a point if and only if the cited conditions are met. To see the second one, since $D\left(l_{1}, l_{2}\right) \subset D\left(l_{1}\right)$, we must deform $D\left(l_{1}, l_{2}\right)$ in a direction normal to $D\left(l_{1}\right)$ so as to become transverse, and then compute the intersection number. But we may deform $D\left(l_{1}, l_{2}\right)$ within $D\left(l_{2}\right)$, as this $i s$ transverse to $D\left(l_{1}\right)$. The intersection number is then seen to be the self-intersection of the curve $D\left(l_{1}, l_{2}\right)$ lying on the surface $D\left(l_{2}\right)$, and, as we have already mentioned, this is -2 . 
Lemma 3.7.

$$
H(\Delta) \cdot D\left(l_{1}, l_{2}\right)=\left\{\begin{array}{c}
1 \quad \text { if } l_{1} \in \delta \text { and } l_{2} \in \delta^{\perp} \text { or vice versa, } \\
\text { where } \Delta=\left\{\delta, \delta^{\perp}\right\}, \\
0 \quad \text { otherwise. }
\end{array}\right.
$$

Proof. $D(l) \cap H(\Delta) \neq \emptyset \Leftrightarrow l \in \delta$ or $l \in \delta^{\perp}$, and that the intersection is of the form cusp $\times \mathcal{A}_{1}(n)^{*}$ or $\mathcal{A}_{1}(n)^{*} \times$ cusp. If both $l_{1}$ and $l_{2}$ were in, say, $\delta$, then they would both be of the form, say, cusp $\times \mathcal{A}_{1}(n)^{*}$, in which case they would be "parallel" or equal. But the intersection number would be 0 in both cases, which is clear. The only other possibility is that $l_{1}$ and $l_{2}$ are on opposite sides of $\Delta$, and the intersection is transverse in one point.

Lemma 3.8.

$$
D\left(l^{\prime}\right) \cdot S(l, \Delta)=\left\{\begin{array}{r}
1 \quad \text { if } l \in \delta \text { and } l^{\prime} \in \delta^{\perp} \text { or vice versa, } \\
\text { where } \Delta=\left\{\delta, \delta^{\perp}\right\} \\
0 \quad \text { otherwise. }
\end{array}\right.
$$

Proof. The first condition states that $S(l, \Delta)=D(l) \cap H(\Delta)$ is cusp $\times \mathcal{A}_{1}(n)^{*}$ and that $D\left(l^{\prime}\right) \cap H(\Delta)$ is of the form $\mathcal{A}_{1}(n)^{*} \times c u s p$, so the intersection is clearly 1 . The only other possibility for a nonzero intersection is for $l=l^{\prime} \in \delta$, say. We must deform $S(l, \Delta)$ in a direction normal to $D(l)$. But we can deform $S(l, \Delta)$ inside $H(\Delta)$, which is transverse to $D(l)$. Thus, we are computing the self-intersection of the curve $S(l, \Delta)$ on the surface $H(\Delta)$; but this is 0 .

\section{Lemma 3.9.}

$$
H\left(\Delta^{\prime}\right) \cdot S(l, \Delta)= \begin{cases}-\mu(n) / 12 & \text { if } \Delta=\Delta^{\prime}, \\ 0 & \text { otherwise. }\end{cases}
$$

Proof. If $\Delta \neq \Delta^{\prime}$ then $H(\Delta) \cap H\left(\Delta^{\prime}\right)=\emptyset$ as we have remarked, so that $S(l, \Delta) \cap$ $H\left(\Delta^{\prime}\right)$ is empty. Otherwise, since $S(l, \Delta) \subset H(\Delta)$, we must deform $S(l, \Delta)$ in a direction normal to $H(\Delta)$. But we can deform it inside $D(l)$, which is transverse to $H(\Delta)$, so we are computing the self-intersection of the curve $S(l, \Delta)$ on the surface $D(l)$, and this we have seen is $-\mu(n) / 12$ (proposition 3.2).

\section{Lemma 3.10.}

$$
D(l) \cdot F\left(l^{\prime}\right)= \begin{cases}-2 n & \text { if } l=l^{\prime}, \\ 0 & \text { otherwise. }\end{cases}
$$

Proof. If $l \neq l^{\prime}$, then $D(l)$ will intersect $D\left(l^{\prime}\right)$, if at all, in a $\mathbf{P}^{1}$ lying over a cusp. We can deform $F\left(l^{\prime}\right)$ so as to be disjoint from any of these. If $l=l^{\prime}$, we observe that $F(l)$ is homologous to the cycle of $\mathbf{P}^{1}$ 's lying over any cusp, so the intersection to be computed is a sum of $n$ terms of the form $D(l) \cdot D\left(l, l_{1}\right)$, each of which is -2 by lemma 3.6 .

\section{Lemma 3.11.}

$$
H(\Delta) \cdot F(l)= \begin{cases}1 & \text { if } l \in \delta \text { or } l \in \delta^{\perp} \text { for } \Delta=\left\{\delta, \delta^{\perp}\right\} \\ 0 & \text { otherwise. }\end{cases}
$$

Proof. $H(\Delta) \cap D(l) \neq \emptyset$ iff $l \in \delta$ or $l \in \delta^{\perp}$, in which case it is one of the $n^{2}$ sections on $D(l)$. This clearly has intersection 1 with a fiber. 


\section{4. (Co)homology of $\mathcal{A}_{2}(3)^{*}$}

In this section we investigate the (co)homology of $\mathcal{A}_{2}(3)^{*}$. We begin by determining the (co)homology groups. We then consider divisors on $\mathcal{A}_{d}(n)^{*}$, and specialize the discussion to obtain explicit algebraic cycles which generate the (co)homology of $\mathcal{A}_{2}(3)^{*}$.

Theorem 4.1. 1. $\mathcal{A}_{2}(3)^{*}$ is simply connected and its integral homology is torsionfree.

2. $\mathrm{H}_{i}\left(\mathcal{A}_{2}(3)^{*}, \mathbf{Z}\right)$ has rank $1,0,61,0,61,0,1$ for $i=0, \ldots, 6$.

Proof. 1) Recall that a smooth projective rational variety $V$ is simply connected. (Any modification occurs on a subvariety $W$ of complex codimension $\geq 1$; then $\pi_{1}(V-W) \rightarrow \pi_{1}(V)$ is an isomorphism if codim $W>1$, and an epimorphism if codim $W=1$, by general position, and of course, $\pi_{1}\left(\mathbf{P}^{n}\right)=0$.) Also, recall that the torsion subgroup of $\mathrm{H}_{2}(V, \mathbf{Z})$ is a birational invariant. This is a theorem of Artin and Mumford 11. These results, together with the universal coefficient theorem, show that (as observed in [1]) any rational 3 -fold is simply connected with torsion-free homology. We observed that $\mathcal{A}_{2}(3)^{*}=B$, the Burkhardt quartic, is rational.

2) In theorem 2.11 we found the zeta function $Z\left(B \otimes \mathbf{F}_{q} / \mathbf{F}_{q}, u\right)$, where $q$ is a prime power $\equiv 1 \bmod 3$. By the Weil conjectures,

$$
Z\left(B \otimes \mathbf{F}_{q} / \mathbf{F}_{q}, u\right)=\prod_{i=0}^{6} P_{i}(u)^{(-1)^{i+1}},
$$

where

$$
P_{i}(u)=\operatorname{det}\left(1-u F \mid \mathrm{H}_{e t}^{i}\left(B \otimes \overline{\mathbf{F}}_{q}, \mathbf{Q}_{l}\right)\right)
$$

is the determinant of the Frobenius acting on the $l$-adic étale cohomology, $l$ being any prime not dividing $q$. Since the eigenvalues of the polynomial $P_{i}(u)$ are of complex absolute value $q^{i / 2}$, there is no cancellation among these various factors in the expression of the zeta function, so we conclude from theorem 2.11 that for our variety $B$ the polynomials are of degrees $1,0,61,0,61,0,1$ for $i=0, \ldots, 6$. On the other hand, the comparison theorem gives

$$
\operatorname{dim}_{\mathbf{C}} \mathrm{H}^{i}(B(\mathbf{C}), \mathbf{C})=\operatorname{dim}_{\mathbf{Q}_{l}} \mathrm{H}_{e t}^{i}\left(B \otimes \overline{\mathbf{F}}_{q}, \mathbf{Q}_{l}\right)=\text { degree } P_{i}(u),
$$

which shows the ranks of these groups, and then the first part of this theorem yields the integral (co)homology of $\mathcal{A}_{2}(3)^{*}$. This argument only requires that $B$ is isomorphic with $\mathcal{A}_{2}(3)^{*}$ over the complex numbers.

Remark 4.2. Heidrich and Knöller [20] have shown that $\pi_{1}\left(\mathcal{A}_{d}(n)^{*}\right)=0$ for $d \geq 2$ and $n$ arbitrary. Of course, these varieties are not in general rational (for example, $\mathcal{A}_{2}(n)^{*}$ is of general type if $n \geq 4$ by calculations of Yamazaki [59]).

Proposition 4.3. Let $d \geq 2$ and $n$ be arbitrary. Set $N=d(d+1)$. Let $[D(l)]$ denote the image of the fundamental class of $\mathrm{H}_{N-2}(D(l), \mathbf{Q})$ in $\mathrm{H}_{N-2}\left(\mathcal{A}_{d}(n)^{*}, \mathbf{Q}\right)$ under the inclusion

$$
D(l) \hookrightarrow \mathcal{A}_{d}(n)^{*} .
$$

Then, $\{[D(l)]\}$ is a linearly independent set in $\mathrm{H}_{N-2}\left(\mathcal{A}_{d}(n)^{*}, \mathbf{Q}\right)$. 
Proof. By Alexander duality,

$$
\mathrm{H}_{N-1}\left(\mathcal{A}_{d}(n)^{*}, \partial \mathcal{A}_{d}(n)^{*}\right)=\mathrm{H}^{1}\left(\mathcal{A}_{d}(n)\right)=\mathrm{H}^{1}\left(\Gamma_{d}(n)\right),
$$

and this group is 0 by Kazhdan's theorem 30 . Thus, by the exact sequence of the pair $\left(\mathcal{A}_{d}(n)^{*}, \partial \mathcal{A}_{d}(n)^{*}\right)$, the map

$$
\mathrm{H}_{N-2}\left(\partial \mathcal{A}_{d}(n)^{*}\right) \longrightarrow \mathrm{H}_{N-1}\left(\mathcal{A}_{d}(n)^{*}\right)
$$

is an injection. But $\partial \mathcal{A}_{d}(n)^{*}$ is a divisor with normal crossings whose distinct boundary components $D(l)$ either intersect in subvarieties of complex codimension 1 , or are disjoint; so, by Mayer-Vietoris,

$$
\mathrm{H}_{N-2}\left(\partial \mathcal{A}_{d}(n)^{*}\right)=\bigoplus_{l} \mathrm{H}_{N-2}(D(l)),
$$

with the summands on the right-hand side generated by $[D(l)]$.

Proposition 4.4. Let $n$ be arbitrary. Let $[H(\Delta)]$ denote the image of the fundamental class of $\mathrm{H}_{4}(H(\Delta), \mathbf{Q})$ in $\mathrm{H}_{4}\left(\mathcal{A}_{2}(n)^{*}, \mathbf{Q}\right)$ under the inclusion

$$
H(\Delta) \hookrightarrow \mathcal{A}_{2}(n)^{*} .
$$

Then $\{[H(\Delta)]\}$ is a linearly independent set in $\mathrm{H}_{4}\left(\mathcal{A}_{2}(n)^{*}, \mathbf{Q}\right)$.

Proof. It suffices to find complex curves $\left\{h_{\Delta}\right\}$ in $\mathcal{A}_{2}(n)^{*}$ with $H(\Delta) \cdot h_{\Delta} \neq 0$, but $H\left(\Delta^{\prime}\right) \cdot h_{\Delta}=0$ if $\Delta \neq \Delta^{\prime}$. By lemma 3.9] we may choose $h_{\Delta}=S(l, \Delta)$.

Theorem 4.5. The 85 classes $[D(l)]$ and $[H(\Delta)]$ span $\mathrm{H}_{4}\left(\mathcal{A}_{2}(3)^{*}, \mathbf{Q}\right)$.

Proof. By propositions 4.3 and 4.4 the classes $[D(l)]$ and $[H(\Delta)]$ span subspaces of dimensions 40 and 45 respectively. Denote these spaces by $\mathcal{D}$ and $\mathcal{H}$ respectively. Then, as a representation space of $G=\mathbf{P S p}(4, \mathbf{Z} / 3)$ the space $\mathcal{D}$ is isomorphic to the rational vector space spanned by $\{l\}$. This is the induced representation of the trivial character from the stabilizing subgroup of any one of the $l$. We can read off this representation from the Atlas of Finite Groups [10. In the notation of the Atlas, it is $1+15 a+24 a$. Similarly, as a representation space of $G$, the space $\mathcal{H}$ is isomorphic to the rational vector space spanned by the $\{\Delta\}$, which is $1+20 a+$ $24 a$. In each case, 1 denotes the trivial 1-dimensional representation. Clearly, this summand in $\mathcal{D}$ is generated by $\sum_{l}[D(l)]$ and this summand in $\mathcal{H}$ is generated by $\sum_{\Delta}[H(\Delta)]$.

Once we show that these two classes are linearly independent, it follows immediately from Schur's lemma that the span of $\mathcal{D} \cup \mathcal{H}$ is either 61 - or 85 -dimensional. Since $\operatorname{dim}_{\mathbf{Q}} \mathrm{H}_{4}\left(\mathcal{A}_{2}(3)^{*}, \mathbf{Q}\right)=61$, it must be the former, and $\mathcal{D} \cup \mathcal{H}$ spans this vector space.

Note that $\sum_{l}[F(l)]$, the homology class represented by the fundamental cycle of

$$
\bigcup_{l} F(l)
$$

is a $G$-invariant class in $\mathrm{H}_{4}\left(\mathcal{A}_{2}(3)^{*}, \mathbf{Q}\right)$, as is $\sum_{l, \Delta}[S(l, \Delta)]$, the homology class represented by the fundamental cycle of

$$
\bigcup_{l, \Delta} S(l, \Delta)
$$


We compute intersection numbers:

$$
\sum_{l}[D(l)] \cdot \sum_{l^{\prime}}\left[F\left(l^{\prime}\right)\right]=40 \cdot(-6)=-240
$$

by lemma 3.10 ,

$$
\sum_{\Delta}[H(\Delta)] \cdot \sum_{l}[F(l)]=45 \cdot 8=360
$$

by lemma 3.11 (as each $\Delta$ contains 8 l's),

$$
\sum_{l^{\prime}}\left[D\left(l^{\prime}\right)\right] \cdot \sum_{l, \Delta}[S(l, \Delta)]=\sum_{\Delta} \sum_{l, l^{\prime}}\left[D\left(l^{\prime}\right)\right] \cdot[S(l, \Delta)]=40 \cdot 8=320
$$

by lemma 3.8 , and

$$
\sum_{\Delta^{\prime}}\left[H\left(\Delta^{\prime}\right)\right] \cdot \sum_{l, \Delta}[S(l, \Delta)]=\sum_{\Delta}[H(\Delta)] \cdot \sum_{l}[S(l, \Delta)]=45 \cdot 8 \cdot(-1)=-360
$$

by lemma 3.9 (noting that $\mu(3)=12$ ).

Then the matrix of these intersections is

$$
\left(\begin{array}{cc}
-240 & 320 \\
360 & -360
\end{array}\right)
$$

which is nonsingular (over $\mathbf{Q}$ ), showing that $\sum_{l}[D(l)]$ and $\sum_{\Delta}[H(\Delta)]$ are independent, as required.

Corollary 4.6. As representation spaces of $G, \mathrm{H}_{i}\left(\mathcal{A}_{2}(3)^{*}, \mathbf{Q}\right)$ are isomorphic to $1+1+15 a+20 a+24 a$ (in the notation of [10]) for $i=2,4$.

Proof. For $i=4$ this is immediate from theorem 4.5 It follows for $i=2$ as the representations of $G$ on $\{l\}$ and $\{\Delta\}$, being permutation representations, are self-dual.

Remark 4.7. The group $G=\mathbf{P S p}(4, \mathbf{Z} / 3)$ acts on $\mathcal{A}_{2}(3)^{*}$ with quotient $\mathcal{A}_{2}(1)^{*}$. In fact the compactification $\mathcal{A}_{2}(3)^{*}$ is equivariant with respect to the action of $G$. By this we mean that the action of $G$ on $\mathcal{A}_{2}(3)$ extends to an action of $G$ on $\mathcal{A}_{2}(3)^{*}$ with quotient $\mathcal{A}_{2}(1)^{*}$. Furthermore, this action of $G$ is compatible with its action on the indexing sets of the boundary components and Humbert surfaces, where the action on indexing sets arises from the left action of $G$ on $V=(\mathbf{Z} / n)^{4}$ given by $g(v)=v \cdot g^{-1}$ ( $v$ being regarded as a row vector). The argument in the above theorem showing the independence of the two $G$-invariant classes gives a new proof of the known fact that

$$
\mathrm{H}_{4}\left(\mathcal{A}_{2}(1)^{*}, \mathbf{Q}\right)=\mathrm{H}_{4}\left(\mathcal{A}_{2}(3)^{*}, \mathbf{Q}\right)^{G}
$$

is 2-dimensional (see [32], 33]). Alternatively, that result could be used to prove the independence.

Remark 4.8. The above theorem shows that the module of homology relations among the 40 boundary components and 45 Humbert surfaces is the unique 24dimensional irreducible representation of the group $G=\mathbf{P S p}(4, \mathbf{Z} / 3)$, and therefore this representation is defined over $\mathbf{Q}$. This is one of the 6 unipotent representations of $G$, and we have given a geometric construction of it. Note also that the other characters $20 a$ and $15 a$ must also be defined over $\mathbf{Q}$ because $1+15 a+24 a$ is and $1+20 a+24 a$ is, both being permutation representations. These characters can be identified with the list made by Srinivasan [53] as follows: $15 a=\theta_{12}, 20 a=$ 
$\chi_{6}(1), 24 a=\theta_{9}$. They can also be described in Deligne-Lusztig theory as follows: We have a decomposition

$$
\mathrm{R}_{T_{0}}^{G}(1)=\operatorname{Ind}_{B}^{G}(1)=1+15 a+15 b+2 \cdot 24 a+81 a,
$$

where $T_{0}$ is the maximal split torus of $G$, and $B$ is a Borel subgroup. This gives 5 of the 6 unipotent characters, the sixth being the character 6 , which is both cuspidal and unipotent. (There is one other cuspidal representation of $G, 64 a$.) Let $T_{s}$ be the maximal nonsplit torus of $\mathbf{G L}(2)$, whose $\mathbf{F}_{3}$ points are $\mathbf{F}_{9}^{\times}$. Let $\omega$ be a character of order 4 of $\mathbf{F}_{9}^{\times}$. The character $-\mathrm{R}_{T_{s}}^{\mathrm{GL}(2)}(\omega)$ is a cuspidal representation of $\mathbf{G L}(2)$, and, regarded as a representation of $L=\mathbf{P G L}\left(2, \mathbf{F}_{3}\right) \cong S_{4}$, it is the unique 2-dimensional irreducible representation of that group. Since $\mathbf{P G L}\left(2, \mathbf{F}_{3}\right)$ is the Levi component of the parabolic $P(h)$ stabilizing an isotropic line, we may consider this also as a character of $P(h)$. Then, a computer calculation using the character table from the Atlas shows that

$$
-\mathrm{R}_{T_{s}}^{G}(\omega)=-\operatorname{Ind} \underset{P(h)}{G}\left(\mathrm{R}_{T}^{L}(\omega)\right)=20 a+60 a,
$$

where we have used well-known properties of the Deligne-Lusztig functors, and we have regarded $T_{s}$ as a maximal torus of $G$ as well. This shows how the character $20 a$ arises in that formalism; the character $60 a$ also appears in the cohomology of the arithmetic group $\Gamma_{2}(3)$, as will be seen. (For a discussion of this theory see [14], especially Chapter 15).

We wish to refine theorem 4.5 to obtain a result valid over the integers. To this end we first examine the Humbert surfaces and boundary components more precisely.

Let us fix $\Delta=\left\{\delta, \delta^{\perp}\right\}$. As we have observed, $H(\Delta) \simeq \mathcal{A}_{1}(3)^{*} \times \mathcal{A}_{1}(3)^{*} \simeq$ $\mathbf{P}^{1} \times \mathbf{P}^{1}$. Furthermore, for $l \in \delta$ (resp. $\left.l^{\prime} \in \delta^{\perp}\right), S(l, \Delta) \subset H(\Delta)$ is of the form $\mathbf{P}^{1} \times \operatorname{cusp}\left(\right.$ resp. $S\left(l^{\prime}, \Delta\right) \subset H(\Delta)$ is of the form cusp $\left.\times \mathbf{P}^{1}\right)$. Note that the curves $S(l, \Delta)$ (resp. $S\left(l^{\prime}, \Delta\right)$ ) are mutually homologous. Actually, $\Delta$ is unordered, i.e., $\left\{\delta, \delta^{\perp}\right\}=\left\{\delta^{\perp}, \delta\right\}$, so there is no canonical way to distinguish these classes. Thus we let

$$
\left\{h_{1}(\Delta), h_{2}(\Delta)\right\}=\left\{S(l, \Delta), S\left(l^{\prime}, \Delta\right)\right\},
$$

a pair of generators of $\mathrm{H}_{2}(H(\Delta), \mathbf{Z})$. Because of this ambiguity, we will always refer to the set $\left\{h_{1}(\Delta), h_{2}(\Delta)\right\}$ and never to one of the individual elements.

Now let us fix $l$. As we have observed, $D(l)$ is the blow-up of $\mathbf{P}^{2}$ in the 9 base-points of the Hesse pencil (and the blow-ups of these points are the 9 sections $S(l, \Delta))$. We let $d(l)$ be the total transform of the hyperplane section under this blow-up. We may choose this hyperplane $\mathbf{P}^{1} \subset \mathbf{P}^{2}$ to be a line through 3 of the base-points in this pencil; then $d(l)$ is a union of 4 lines. More precisely, choose an $h$ with $h=l \wedge l^{\prime}$ for some $l^{\prime}$. There are three values $\Delta_{1}, \Delta_{2}, \Delta_{3}$ of $\Delta$ for which $D\left(l, l^{\prime}\right) \cap S(l, \Delta)$ is non-empty, in which case it is a point. This happens when $\Delta_{i}=\left\{\delta, \delta^{\perp}\right\}$ with $l \in \delta$ and $l^{\prime} \in \delta^{\perp}$. Then

$$
[d(l)]=\left[D\left(l, l^{\prime}\right)\right]+\sum_{i}\left[S\left(l, \Delta_{i}\right)\right]
$$

in $\mathrm{H}_{2}(D(l), \mathbf{Z})$. Note that $D\left(l, l^{\prime}\right)$ is one line in a 3-gon, and $D\left(l, l^{\prime \prime}\right) \cap S\left(l, \Delta_{i}\right)=\emptyset$, $i=1,2,3$, for $D\left(l, l^{\prime \prime}\right)$ any of the other lines in the 3 -gon, i.e. for $l^{\prime \prime} \neq l^{\prime}$ but $h=l \wedge l^{\prime \prime}$. On the other hand, if $h^{\prime}=l \wedge l^{\prime \prime}, h^{\prime} \neq h$, then $D\left(l, l^{\prime \prime}\right) \cap S\left(l, \Delta_{i}\right) \neq \emptyset$, in which case it is a point, for exactly one value of $i \in\{1,2,3\}$ (Note that there are 
then 3 choices for $l^{\prime \prime}$ and the $D\left(l, l^{\prime}\right)$ then form a 3-gon). Notice also that a different choice of $l^{\prime}$ will yield a different cycle representing $[d(l)]$, but this homology class is independent of the choice.

Theorem 4.9. 1 . The 85 classes $[H(\Delta)]$ and $[D(l)]$ generate $\mathrm{H}_{4}\left(\mathcal{A}_{2}(3)^{*}, \mathbf{Z}\right)$.

2. The 130 classes $\left\{h_{1}(\Delta), h_{2}(\Delta)\right\},\{d(l)\}$ generate $\mathrm{H}_{2}\left(\mathcal{A}_{2}(3)^{*}, \mathbf{Z}\right)$.

Proof. We prove parts 1) and 2) by calculating the 85 by 130 intersection matrix of these two sets:

a) $H(\Delta) \cdot h_{i}\left(\Delta^{\prime}\right)=-1$ if $\Delta=\Delta^{\prime}$ and $i=1,2$, and $=0$ if $\Delta \neq \Delta^{\prime}$.

This follows immediately from lemma 3.9, remembering that $\left\{h_{1}(\Delta), h_{2}(\Delta)\right\}=$ $\left\{S(l, \Delta), S\left(l^{\prime}, \Delta\right)\right\}$ for suitable $l, l^{\prime}$.

b) $D(l) \cdot h_{i}(\Delta)=1$ if $h_{i}(\Delta)=S\left(l^{\prime}, \Delta\right)$ for some $l^{\prime}$ with $l \in \delta l^{\prime} \in \delta^{\perp}$, where $\Delta=\left\{\delta, \delta^{\perp}\right\}$, and $=0$ otherwise.

This is immediate from lemma 3.8.

c) $H(\Delta) \cdot d(l)=0$.

There are three cases to consider. If $l \notin \Delta$, so that $H(\Delta) \cap D(l)=\emptyset$, this is certainly true. Suppose $l \in \Delta$. If $\Delta \notin\left\{\Delta_{1}, \Delta_{2}, \Delta_{3}\right\}$ (the $\Delta_{i}$ chosen as in the definition of $d(l))$, then $H(\Delta) \cap S\left(l, \Delta_{i}\right)=\emptyset, i=1,2,3$, and $H(\Delta) \cap D\left(l, l^{\prime}\right)=\emptyset$, so again the intersection number is 0 . Finally, if $\Delta=\Delta_{1}$ (and similarly for $\Delta_{2}, \Delta_{3}$ ), then

$$
H(\Delta) \cdot d(l)=H(\Delta) \cdot D\left(l, l^{\prime}\right)+H(\Delta) \cdot S(l, \Delta)=1+(-1)=0
$$

by lemmas 3.7 and 3.9

d) $D\left(l^{\prime}\right) \cdot d(l)=-2$ if $l^{\prime}=l$; $=1$ if $h=l \wedge l^{\prime}$ for some $h$; and $=0$ otherwise.

If $l^{\prime}=l$ this is immediate from lemmas 3.6 and 3.8 If $h=l \wedge l^{\prime}$, then we may use $l^{\prime}$ to define $d(l)$, so

$$
D\left(l^{\prime}\right) \cdot d(l)=D\left(l^{\prime}\right) \cdot D\left(l, l^{\prime}\right)+\sum_{i} D\left(l^{\prime}\right) \cdot S\left(l, \Delta_{i}\right)=-2+3 \cdot 1=1
$$

by lemmas 3.6 and 3.8 . Otherwise, $D(l) \cap D\left(l^{\prime}\right)=\emptyset$, so the intersection number is certainly 0 .

This gives all the entries of the intersection matrix. Now a (computer) calculation shows that the matrix has rank 61 and that the 61 elementary divisors (over $\mathbf{Z}$ ) are all 1 , yielding the theorem.

Remark 4.10. a) Proposition 1.4 is immediate by duality.

b) Propositions 4.3 and 4.4 imply that each of $\{D(l)\}$ and $\{H(\Delta)\}$ extend to a basis of $\mathrm{H}_{4}\left(\mathcal{A}_{2}(3)^{*}, \mathbf{Q}\right)$. Calculations with the above intersection matrix show that neither set extends to a basis of $\mathrm{H}_{4}\left(\mathcal{A}_{2}(3)^{*}, \mathbf{Z}\right)$.

Corollary 4.11. The Hodge numbers of $\mathcal{A}_{2}(3)^{*}$ are as follows:

$$
\mathrm{h}^{0,0}=1, \quad \mathrm{~h}^{1,1}=61, \quad \mathrm{~h}^{2,2}=61, \quad \mathrm{~h}^{3,3}=1, \quad \mathrm{~h}^{i, j}=0 \text { otherwise }
$$

Proof. All of $\mathrm{H}_{*}\left(\mathcal{A}_{2}(3)^{*}, \mathbf{Q}\right)$ is represented by algebraic cycles.

\section{Cohomology of $\mathcal{A}_{2}(3)$}

We are going to compute the cohomology groups of $\Gamma_{2}(3)$ by using the Leray spectral sequence for the inclusion

$$
j: \mathcal{A}_{2}(n) \hookrightarrow \mathcal{A}_{2}(n)^{*},
$$




$$
\mathrm{E}_{2}^{p, q}=\mathrm{H}^{p}\left(\mathcal{A}_{2}(n)^{*}, \mathrm{R}^{q} j_{*} \mathbf{Q}\right) \Longrightarrow \mathrm{H}^{p+q}\left(\mathcal{A}_{2}(n), \mathbf{Q}\right)
$$

for $n=3$. But first let us recall some general facts for arbitrary $n$, due to Oda and Schwermer [42]. The corresponding sequence was analyzed by Deligne 12 for an arbitrary inclusion of the complement of a divisor with normal crossings $X \hookrightarrow \bar{X}$ on a smooth complete variety $\bar{X}$. It is a theorem of Deligne (loc. cit.) that this spectral sequence degenerates in $\mathrm{E}_{3}$, and the resulting filtration on the abutment is, up to a renumbering, the weight filtration of the mixed Hodge structure on the latter. More precisely,

$$
\mathrm{E}_{3}^{p, q}=\mathrm{E}_{\infty}^{p, q}=\mathrm{Gr}_{p+2 q}^{W} \mathrm{H}^{p+q}(X, \mathbf{Q}) .
$$

Recall also that $\operatorname{Gr}_{i}^{W} \mathrm{H}^{j}(X, \mathbf{Q})=0$ unless $j \leq i \leq 2 j$, and that $\operatorname{Gr}_{i}^{W} \mathrm{H}^{i}(X, \mathbf{Q})$ is the image of

$$
\mathrm{H}^{i}(\bar{X}, \mathbf{Q}) \longrightarrow \mathrm{H}^{i}(X, \mathbf{Q})
$$

for any smooth compactification $\bar{X}$ of $X$. Let

$$
D^{[q]}=\left\{\text { disjoint union of all the } D\left(l_{1}, \ldots, l_{q}\right) \text { for } l_{1}<\cdots<l_{q}\right\} \text {, }
$$

where $D^{[0]}=\mathcal{A}_{2}(n)^{*}$. This is empty if $q>3$. Then

$$
\mathrm{H}^{p}\left(\mathcal{A}_{2}(n)^{*}, \mathrm{R}^{q} j_{*} \mathbf{Q}\right)=\mathrm{H}^{p}\left(D^{[q]}, \varepsilon\right)=\bigoplus_{I_{q}} \mathrm{H}^{p}\left(D\left(l_{1}, \ldots, l_{q}\right), \varepsilon\right),
$$

where $I_{q}$ is the set of $l_{1}<\cdots<l_{q}$ in some arbitrary order of the $l$. The $\varepsilon$ are one-dimensional Q-local systems of the form

$$
\bigwedge^{q} \mathbf{Q}^{E_{q}}
$$

where $E_{q}$ is the sheaf of germs of the $q$ hypersurfaces meeting in $D\left(l_{1}, \ldots, l_{q}\right)$. As Deligne points out in [12, if the divisor at infinity is globally a sum of smooth divisors, as is the case here, then the choice of a total ordering of the $D(l)$ trivializes all these local systems: $\varepsilon \sim \mathbf{Q}$. We will choose such an ordering in this section. However, this identification is not functorial, and in section 6 when we consider the action of the finite group $\mathbf{P S p}\left(4, \mathbf{F}_{3}\right)$ it will be crucial to consider these sheaves. Indeed, as will be seen in the next section, we will examine certain induced representations from the stabilizer subgroups inside $\mathbf{P S p}\left(4, \mathbf{F}_{3}\right)$ of various $D\left(l_{1}, \ldots, l_{q}\right)$. These stabilizer subgroups act nontrivially on the hypersurfaces $D\left(l_{1}\right), \ldots, D\left(l_{q}\right)$ containing the component $D\left(l_{1}, \ldots, l_{q}\right)$, and hence on the corresponding local system $\varepsilon$. This action is expressed via the determinant character of the permutation action on these hypersurfaces, and thus will contribute nontrivially to the character decompositions of the various cohomology spaces.

Theorem 5.1 ([42]). The map

$$
\mathrm{H}^{2}\left(\mathcal{A}_{2}(n)^{*}, \mathbf{Q}\right) \longrightarrow \mathrm{H}^{2}\left(\mathcal{A}_{2}(n), \mathbf{Q}\right)
$$

is onto. In other words, $\mathrm{H}^{2}\left(\mathcal{A}_{2}(n), \mathbf{Q}\right)$ is pure of weight 2.

This computes the second Betti number of $\mathcal{A}_{2}(n)$ from that of $\mathcal{A}_{2}(n)^{*}$ because of the lemma below:

Lemma 5.2. The map

$$
\mathrm{E}_{2}^{0,1}=\bigoplus_{l} \mathrm{H}^{2}(D(l), \mathbf{Q}) \longrightarrow \mathrm{E}_{2}^{2,0}=\mathrm{H}^{2}\left(\mathcal{A}_{2}(n)^{*}, \mathbf{Q}\right)
$$


is injective. Therefore

$$
\operatorname{dim} \mathrm{H}^{2}\left(\mathcal{A}_{2}(n), \mathbf{Q}\right)=\operatorname{dim} \mathrm{H}^{2}\left(\mathcal{A}_{2}(n)^{*}, \mathbf{Q}\right)-\# \mathfrak{P}_{1}(\mathbf{Z} / n) .
$$

Proof. The constituent maps $\mathrm{H}^{2}(D(l), \mathbf{Q}) \rightarrow \mathrm{H}^{2}\left(\mathcal{A}_{2}(n)^{*}, \mathbf{Q}\right)$ carry 1 to the cycle class of the divisor $D(l) \subset \mathcal{A}_{2}(n)^{*}$, and it is to be shown that these are Qhomologically independent. This follows from the dual of proposition 4.3

Since $\operatorname{Gr}_{i}^{W} \mathrm{H}^{2}=0$ for $i=3$, we see that $\mathrm{E}_{2}^{1,1} \longrightarrow \mathrm{E}_{2}^{3,0}$ is injective, and hence

$$
\operatorname{dim} \mathrm{Gr}_{3}^{W} \mathrm{H}^{3}\left(\mathcal{A}_{2}(n), \mathbf{Q}\right)=\operatorname{dim} \mathrm{H}^{3}\left(\mathcal{A}_{2}(n)^{*}, \mathbf{Q}\right)-\sum_{l} \operatorname{dim} \mathrm{H}^{1}(D(l), \mathbf{Q}) .
$$

We introduce the complexes

$$
\begin{array}{lllll}
S^{\bullet} & : & \mathrm{E}_{2}^{0,2} \stackrel{d_{S}^{1}}{\longrightarrow} \mathrm{E}_{2}^{2,1} \stackrel{d_{S}^{2}}{\longrightarrow} \mathrm{E}_{2}^{4,0}, \\
T^{\bullet}: & \mathrm{E}_{2}^{0,3} \stackrel{d_{T}^{1}}{\longrightarrow} \mathrm{E}_{2}^{2,2} \stackrel{d_{T}^{2}}{\longrightarrow} \mathrm{E}_{2}^{4,1} \stackrel{d_{T}^{3}}{\longrightarrow} \mathrm{E}_{2}^{6,0} .
\end{array}
$$

$d_{S}^{1}$ is injective since $\operatorname{Gr}_{i}^{W} \mathrm{H}^{2}=0$ for $i=4$. Also, $T^{\bullet}$ is exact in $T^{2}$ and $T^{3}$, because $\mathrm{H}^{i}\left(\mathcal{A}_{2}(n), \mathbf{Q}\right)=0$ for $i \geq 5$. We have

Theorem $5.3([4])$.

$$
\begin{aligned}
& \operatorname{Gr}_{i}^{W} \mathrm{H}^{2}\left(\mathcal{A}_{2}(n), \mathbf{Q}\right)= \begin{cases}\mathrm{H}^{2}\left(\mathcal{A}_{2}(n)^{*}, \mathbf{Q}\right) / \oplus \mathrm{H}^{0}(D(l), \mathbf{Q}) & \text { for } i=2, \\
0 & \text { otherwise, }\end{cases} \\
& \operatorname{Gr}_{i}^{W} \mathrm{H}^{3}\left(\mathcal{A}_{2}(n), \mathbf{Q}\right)= \begin{cases}\mathrm{H}^{3}\left(\mathcal{A}_{2}(n)^{*}, \mathbf{Q}\right) / \oplus \mathrm{H}^{1}(D(l), \mathbf{Q}) & \text { for } i=3, \\
\mathrm{H}^{1}(S) & \text { for } i=4, \\
0 & \text { for } i=5, \\
\mathrm{H}^{0}(T) & \text { for } i=6, \\
0 & \text { otherwise, }\end{cases} \\
& \operatorname{Gr}_{i}^{W} \mathrm{H}^{4}\left(\mathcal{A}_{2}(n), \mathbf{Q}\right)= \begin{cases}\mathrm{H}^{2}(S) & \text { for } i=4, \\
\oplus \mathrm{H}^{3}(D(l), \mathbf{Q}) & \text { for } i=5, \\
\mathrm{H}^{1}(T) & \text { for } i=6, \\
0 & \text { otherwise. }\end{cases}
\end{aligned}
$$

Each differential

$$
d: \bigoplus \mathrm{H}^{p}\left(D\left(l_{1}, \ldots, l_{q}\right), \mathbf{Q}\right) \longrightarrow \bigoplus \mathrm{H}^{p+2}\left(D\left(l_{1}, \ldots, l_{q-1}\right), \mathbf{Q}\right)
$$

is the direct sum of terms of the form $\sum(-1)^{i} d_{i *}$, where $d_{i *}$ is the Gysin homomorphism associated to the inclusion

$$
d_{i}: D\left(l_{1}, \ldots, l_{q}\right) \longrightarrow D\left(l_{1}, \ldots, \widehat{l_{i}}, \ldots, l_{q-1}\right) .
$$

These Gysin maps are easy to understand when applied to cohomology classes of algebraic cycles. If $x$ is such a class, associated to a codimension $r$ cycle on $D\left(l_{1}, \ldots, l_{q}\right)$, then $d_{i *}(x)$ is the class of that same cycle, but now of codimension $r+1$ on

$$
D\left(l_{1}, \ldots, \widehat{l_{i}}, \ldots, l_{q}\right)
$$

The above theorem shows that to compute the Betti numbers of $\Gamma_{2}(n)$, one needs to compute the cohomology of the Igusa compactification and the ranks of the operators $d_{S}^{i}, d_{T}^{i}$. The ranks of $d_{S}^{1}, d_{T}^{2}, d_{T}^{3}$ are known, as we have already mentioned. We can determine the rank of $d_{T}^{1}$ : 
Lemma 5.4. $\operatorname{dim} \operatorname{Ker}\left(d_{T}^{1}\right)=\# \mathfrak{P}_{2}(\mathbf{Z} / n)$.

Proof. By disjointness of the $C(h)$ for various $h$, we see that

$$
d_{T}^{1}=\bigoplus_{h} d_{h}
$$

is a direct sum over those $h$; namely, we look at the subcomplex associated to the $l \in \mathfrak{P}_{1}(\mathbf{Z} / n)$ that are incident upon each $h$. These complexes have the form

$$
d_{h}: \bigoplus \mathbf{Q} e_{i} \longrightarrow \bigoplus \mathbf{Q} f_{j}
$$

where the $e_{i}$ (resp. the $f_{j}$ ) are the 0-cells (resp. the 1-cells) for the 1-skeleton of the tessellation of the Riemann surface that was discussed in section 3.1. In particular, $\mathrm{H}^{0}\left(d_{h}\right)$ is spanned by the connected components; but this 1-skeleton is connected, so each of these is 1-dimensional.

In analyzing the differential $d=d_{S}^{2}$, first observe that these Gysin homomorphisms are morphisms of type $(1,1)$ for the Hodge decompositions. We can therefore decompose

$$
\begin{gathered}
d=d_{\mathrm{alg}}+d_{\mathrm{tr}}+\bar{d}_{\mathrm{tr}}, \quad \text { where } \quad \bar{d}_{\mathrm{tr}} \text { is the complex conjugate of } d_{\mathrm{tr}}, \\
d_{\mathrm{alg}}: \bigoplus_{l} \mathrm{H}^{1,1}(D(l)) \longrightarrow \bigoplus_{l} \mathrm{H}^{2,2}\left(\mathcal{A}_{2}(n)^{*}\right), \\
d_{\mathrm{tr}}: \bigoplus_{l} \mathrm{H}^{2,0}(D(l)) \longrightarrow \mathrm{H}^{3,1}\left(\mathcal{A}_{2}(n)^{*}\right) .
\end{gathered}
$$

Proposition 5.5. Suppose that $\mathrm{H}^{1,1}\left(\mathcal{A}_{2}(n)^{*}\right)$ is generated by the cycle classes of the $D(l)$ and the $H(\Delta)$. Then $d_{\mathrm{alg}}$ is surjective.

Proof. By the Lefschetz isomorphism, cup product with the class of an ample line bundle $L$ gives rise to an isomorphism

$$
L: \mathrm{H}^{2}\left(\mathcal{A}_{2}(n)^{*}, \mathbf{Q}\right) \simeq \mathrm{H}^{4}\left(\mathcal{A}_{2}(n)^{*}, \mathbf{Q}\right),
$$

which is of type $(1,1)$ for the Hodge decompositions. We can take for $L$ the line bundle the sections of whose powers are the Siegel modular forms of various weights. Under the hypothesis, the classes of $L \cdot D(l)$ and $L \cdot H(\Delta)$ generate $\mathrm{H}^{2,2}$, so it will suffice to see that these classes are in the image of $d_{\text {alg. }}$. These classes are the Gysin images of the Chern classes of the restrictions of $L$ to $D(l)$ and $H(\Delta)$. Yamazaki has shown [59] that

$$
\begin{gathered}
L \mid D(l)=\pi^{*} F, \\
L \mid H(\Delta)=\pi_{1}^{*} F \otimes \pi_{2}^{*} F,
\end{gathered}
$$

where $\pi$ (resp. $\pi_{1}, \pi_{2}$ ) is the projection onto the modular curve $M=\mathcal{A}_{1}(n)^{*}$ (resp. are the two projections onto $M$ ), and $F$ is the line bundle on $M$ corresponding to the modular forms of weight one. The classes of the first sort above are certainly in the image of $d_{\text {alg }}$ (for this the explicit form of $L \mid D(l)$ is irrelevant). In the second case, the Chern class of the restricted line bundle is homologically equivalent to a combination of cycles of the forms

$$
\text { cusp } \times M \text { and } M \times \text { cusp, }
$$

and as we have already mentioned, each of these is of the form $H(\Delta) \cap D(l)$. But 
then it is clear that these are in the Gysin image of the inclusion

$$
D(l) \hookrightarrow \mathcal{A}_{2}(n)^{*} .
$$

Remark 5.6. In fact Yamazaki has shown that

$$
10[L]=2 \sum_{\Delta}[H(\Delta)]+n \sum_{\Delta}[D(l)]
$$

in the Picard group of $\mathcal{A}_{2}(n)^{*}$, so it is possible to determine $L \cdot D(l)$ and $L \cdot H(\Delta)$ in terms of the cycles given in section 3.3.

The above discussion of the properties of the spectral sequence has been in the context of $\mathcal{A}_{2}(n)(\mathbf{C})$ for a general level $n$. For $n=3$ the above results suffice to determine all the differentials. Thus we have proved

Theorem 5.7. Let $\mathrm{h}^{p, q}(m)$ denote the dimension of the $(p, q)$-part of the Hodge structure of pure weight

$$
\operatorname{Gr}_{p+q}^{W}\left(\mathrm{H}^{m}\left(\mathcal{A}_{2}(3), \mathbf{C}\right)\right) .
$$

The nonzero ones are

1. $\mathrm{h}^{0,0}(0)=1$,

2. $\mathrm{h}^{1,1}(2)=21$,

3. $\mathrm{h}^{2,2}(3)=99, \mathrm{~h}^{3,3}(3)=40$,

4. $\mathrm{h}^{3,3}(4)=81$.

Theorem 5.8. Let $\mathrm{IH}^{m}\left(\mathcal{A}_{2}(3)^{\text {sa }}, \mathbf{Q}\right)$ denote the intersection cohomology with middle perversity of the Satake compactification. The dimensions are

$$
1,0,21,0,21,0,1
$$

for $m=0, \ldots, 6$, and 0 for all others. The Hodge structures in dimensions 2 and 4 are purely of type $(1,1)$ and (2, 2) respectively.

Proof. After tensoring with $\mathbf{C}$, the intersection cohomology becomes isomorphic with the $\mathrm{L}^{2}$-cohomology

$$
\mathrm{H}_{(2)}^{m}\left(\mathcal{A}_{2}(3), \mathbf{C}\right)
$$

by the solution to Zucker's conjecture given by Looijenga [37], Saper and Stern [46], and Ohsawa [43. Weissauer showed that the natural map

$$
\mathrm{H}_{(2)}^{2}\left(\mathcal{A}_{2}(n), \mathbf{C}\right) \longrightarrow \mathrm{H}^{2}\left(\mathcal{A}_{2}(n), \mathbf{C}\right)
$$

is an isomorphism ([57, Lemma 12]). This proves the claims about $m=2$. We get the results for $m=4$ by Poincaré duality. To get the assertions about $m=1,3,5$, observe that the decomposition theorem [13] shows that the intersection cohomology of the Satake compactification is a direct summand of the cohomology of a resolution of singularities of it; but we have seen that these groups are all 0. Alternatively, we can derive this theorem from the general results of Durfee [15, which depend on the theory of M. Saito [44]. (We thank Daniel Cohen for bringing Durfee's article to our attention.) 


\section{Characters}

The main result of this section is:

Theorem 6.1. As representation spaces of $G=\mathbf{P S p}\left(4, \mathbf{F}_{3}\right)$,

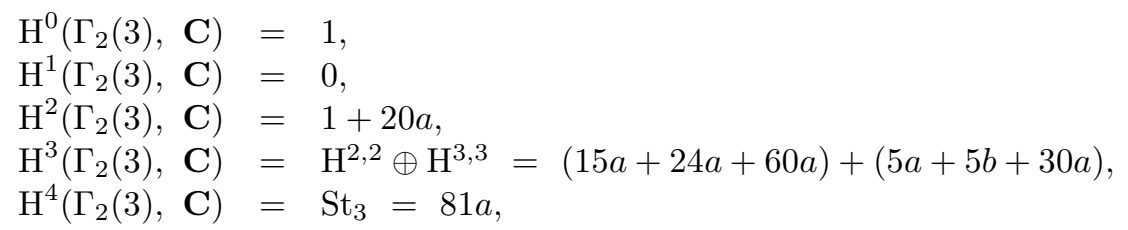

where the integers denote the representations so given in the Atlas [10], and $\mathrm{St}_{3}$ is the Steinberg representation.

We derive this from an analysis of the spectral sequence in section 5 and especially the complexes denoted $S^{\bullet}$ and $T^{\bullet}$ there. The complex $S^{\bullet}$ is

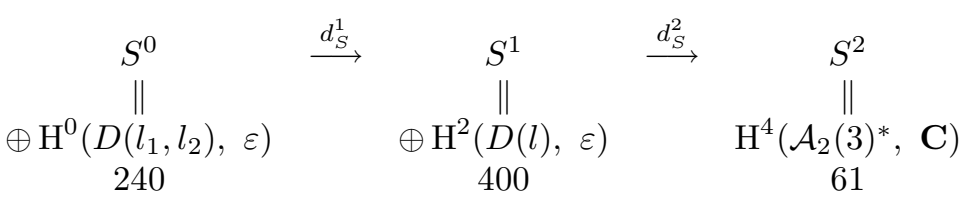

where the dimensions are indicated by the integers below the spaces. The complex $T^{\bullet}$ is

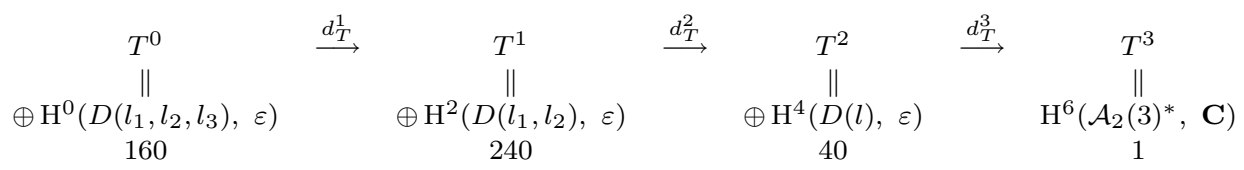

Recall that $D(l)$ is a boundary component, $D\left(l_{1}, l_{2}\right)$ is $\mathbf{P}^{1}(\mathbf{C})$, and $D\left(l_{1}, l_{2}, l_{3}\right)$ is a point. We have denoted by $\varepsilon$ the various one-dimensional local systems of germs of hypersurfaces (see the discussion preceding Theorem 5.1). It is necessary to keep track of these because they contribute signs to the various character spaces.We already know the following dimensions:

$$
\begin{aligned}
& \mathrm{H}^{0}\left(S^{\bullet}\right)=\mathrm{H}^{2}\left(S^{\bullet}\right)=0, \\
& \mathrm{H}^{1}\left(S^{\bullet}\right)=\mathrm{Gr}_{4}^{W} \mathrm{H}^{3}\left(\Gamma_{2}(3)\right)=99, \\
& \mathrm{H}^{2}\left(T^{\bullet}\right)=\mathrm{H}^{3}\left(T^{\bullet}\right)=0 \text {, } \\
& \mathrm{H}^{0}\left(T^{\bullet}\right)=\operatorname{Gr}_{6}^{W} \mathrm{H}^{3}\left(\Gamma_{2}(3)\right)=40 \text {, } \\
& \mathrm{H}^{1}\left(T^{\bullet}\right)=\operatorname{Gr}_{6}^{W} \mathrm{H}^{4}\left(\Gamma_{2}(3)\right)=81 \text {. }
\end{aligned}
$$

We also know that, as representations of $G$ (notation as in the Atlas),

$$
\begin{aligned}
\mathrm{H}^{6}\left(\mathcal{A}_{2}(3)^{*}\right) & =1, \\
\mathrm{H}^{4}\left(\mathcal{A}_{2}(3)^{*}\right) & =1+1+15 a+20 a+24 a, \\
T^{2} & =1+15 a+24 a .
\end{aligned}
$$

It is clear that $S^{0}$ and $T^{1}$ are isomorphic representations. Our main result is

Proposition 6.2. 1. $T^{0}=5 a+5 b+2 \cdot 30 a+45 a+45 b$.

2. $S^{0}=T^{1}=15 a+24 a+30 a+45 a+45 b+81 a$.

3. $S^{1}=1+1+3 \cdot 15 a+20 a+3 \cdot 24 a+30 a+45 a+45 b+60 a+81 a$.

Assuming this proposition, we can prove theorem6.1 as follows: From the known exactness properties of the complexes $S^{\bullet}$ and $T^{\bullet}$ (see the previous section) we obtain 
first of all that $\mathrm{H}^{0}\left(T^{\bullet}\right)$ is 40-dimensional, and so it must be $5 a+5 b+30 a$; and then it follows that $\mathrm{H}^{1}\left(T^{\bullet}\right)$ is $81 a$, the Steinberg representation. One then finds that $\mathrm{H}^{1}\left(S^{\bullet}\right)$ is $15 a+24 a+60 a$. The only nonzero cohomology group of the $S$ complex is $\mathrm{H}^{1}$, which in view of the above is seen to be $15 a+24 a+60 a$, as claimed.

The rest of this section is devoted to the proof of proposition 6.2. All three claims made therein were verified by brute force computer calculations done independently by both authors. However, we present below a more detailed analysis, based in part on the theory of Deligne and Lusztig. This also shows where all the characters that do arise actually come from in that theory.

All the characters to be decomposed are of the form $\operatorname{Ind}_{P}^{G}(\theta)$ for representations $\theta$ of various subgroups $P \subset G$. All the groups $P=P(z)$ are stabilizers of certain geometric configurations $z$ in the 4 -dimensional symplectic vector space over the field with 3 elements, and all of them have a natural semidirect product decomposition

$$
P(z)=U(z) \cdot L(z),
$$

where $U(z)$ is the normal subgroup. Given a representation $\theta: L(z) \longrightarrow \mathbf{G L}(V)$, we let $\hat{\theta}$ be the representation

$$
P(z) \stackrel{\text { projection }}{\longrightarrow} L(z) \stackrel{\theta}{\longrightarrow} \mathbf{G L}(V) .
$$

The following lemma is easily verified and is used implicitly in some of the calculations to follow.

Lemma 6.3. Given $L_{1} \subset L_{2}$ such that $U \cdot L_{1} \subset U \cdot L_{2}$, and a representation $\theta$ of $L_{1}$, we have

$$
\widehat{\operatorname{Ind}_{L_{1}}^{L_{2}}(\theta)}=\operatorname{Ind}_{U \cdot L_{1}}^{U \cdot L_{2}}(\hat{\theta})
$$

Step 1. $T^{0}=\operatorname{Ind}_{P\left(l_{1}, l_{2}, l_{3}\right)}^{G}(\chi)$.

$P\left(l_{1}, l_{2}, l_{3}\right)$ is the stabilizer of $D\left(l_{1}, l_{2}, l_{3}\right)$, which is a point. $l_{1}, l_{2}, l_{3}$ are 3 of the 4 points on an isotropic line $h$, Therefore, $P\left(l_{1}, l_{2}, l_{3}\right)$ may be identified with the stabilizer of $\left(l_{4}, h\right)$, where $l_{4}$ is the fourth point, or in other words of the flag $l_{4} \subset h$. This is exactly the description of ( $\mathbf{F}_{3}$-rational points on) a Borel subgroup $B$ of $G$. The matrices are of the shape

$$
\left(\begin{array}{cc|cc}
t_{1} & * & * & * \\
0 & t_{2} & * & * \\
\hline 0 & 0 & t_{1}^{-1} & 0 \\
0 & 0 & * & t_{2}^{-1}
\end{array}\right)
$$

$P\left(l_{1}, l_{2}, l_{3}\right)$ acts as a full group of permutations on the set $\left\{l_{1}, l_{2}, l_{3}\right\}$, and the local system $\varepsilon$ is just equivalent to a character

$$
\chi: B \longrightarrow \mathrm{S}_{3} \stackrel{\text { sgn }}{\longrightarrow}\{ \pm 1\}
$$

giving the signature of the permutation on the hyperplanes $D\left(l_{1}\right), D\left(l_{2}\right), D\left(l_{3}\right)$. Writing $B=U \cdot T$, where $T$ is the group of diagonal matrices and $U$ consists of the matrices with ones on the diagonal, we see that, $U$ being a 3-group (it is the Sylow 3-subgroup of $B$ ), the character $\chi$ factors through $T$ (the signature of a 3 -cycle is 1 ). Actually it is not difficult to verify that $\chi$ is the determinant character det $: T \rightarrow\{ \pm 1\}$, so the task is to decompose $\operatorname{Ind}_{B}^{G}(\widehat{\operatorname{det}})$. Springer has already carried out this decomposition (or rather, he has determined the irreducible constituents); see [6, the table on p. D-43, the entry corresponding to $\chi_{1}=\chi_{1}=\alpha$,]. He gets characters labelled $\theta_{1}, \theta_{2}, \theta_{3}, \theta_{4}, \Phi_{9}$ in Srinivasan's table, [53]. These are 
the characters $\chi_{16}, \chi_{17}, \chi_{2}, \chi_{3}, \chi_{11}$ of the Atlas. In order to get the pattern of multiplicities claimed, we observe that

$$
\operatorname{Ind}_{B}^{G}(\chi)=R_{T}^{G}(\mathrm{det})
$$

in Deligne-Lusztig theory, and according to the theorem of Howlett and Lehrer, [25], [14, p. 59], the irreducible constituents of this are parameterized by the irreducible characters $\lambda$ of the subgroup $W$ (det) of the Weyl group $W$ that stabilizes the character det. Moreover, the multiplicity of the constituent $\rho_{\lambda}$ associated to $\lambda$ is $\operatorname{dim} \lambda$. $W$ acts on $T$ by $t_{1} \leftrightarrow t_{2}$ and by $t_{i} \rightarrow t_{i}^{ \pm 1}$, but since each $t_{i}= \pm 1$ it is clear that $W(\operatorname{det})=W$. As $W$ is isomorphic with the dihedral group of order 8 , its irreducible representations have dimensions $1,1,1,1,2$. Now there is only one way to attach these multiplicities to the characters in Springer's list to get a dimension of 160 , and this gives part 1 of the proposition. In the same way we can show that

$$
\operatorname{Ind}_{B}^{G}(1)=1+15 a+15 b+2 \cdot 24 a+81 a,
$$

a result to be used below, and easily derivable directly from results in the Atlas.

The type of induction considered here, namely, inducing a cuspidal character from the Levi component of a parabolic, is called Harish-Chandra induction in Digne and Michel's book, and it is for these that the theorem of Howlett and Lehrer applies. One remark here: Springer unfortunately does not indicate how he came up with his table. In fact, the appeal to his results and Deligne-Lusztig theory is not necessary, as we have explicitly decomposed this character by computations on a computer. For this and more information on the characters of this group, see the sequel to this paper [24].

Step 2. $T^{1}=\operatorname{Ind}_{P\left(l_{1}, l_{2}\right)}^{G}(\psi)$.

The subgroup $P\left(l_{1}, l_{2}\right)$ is the stabilizer of $D\left(l_{1}, l_{2}\right)=\mathbf{P}^{1}$, and is described as follows: if an element of $G$ fixes $\left\{l_{1}, l_{2}\right\}$, then it fixes the isotropic line $h$ they span. Therefore $P\left(l_{1}, l_{2}\right) \subset P(h)$, where $P(h)$ is the parabolic which in matrix form has the shape

$$
\left(\begin{array}{c|c}
A & * \\
\hline 0 & { }^{t} A^{-1}
\end{array}\right),
$$

where $A \in \mathbf{P G L}(2, \mathbf{Z} / 3)$. One can see that $P\left(l_{1}, l_{2}\right)$ consists of those matrices whose $A$-component is in the subgroup generated by the diagonal matrices and the flip

$$
\left(\begin{array}{ll}
0 & 1 \\
1 & 0
\end{array}\right) .
$$

Call this group $N(T)$, a "normalizer of a torus". We have

$$
P\left(l_{1}, l_{2}\right)=U(h) \cdot N(T) .
$$

The local system $\varepsilon$ on $P\left(l_{1}, l_{2}\right)$ is given by a character

$$
\psi: P\left(l_{1}, l_{2}\right) \longrightarrow\{ \pm 1\}
$$

which gives -1 precisely when $l_{1}$ and $l_{2}$ are interchanged. Again since $U(h)$ is a 3 -group, this character factors through $N(T)$. Here it is useful to remember the isomorphism $\mathbf{P G L}(2, \mathbf{Z} / 3) \simeq \mathrm{S}_{4}$, which can be realized by the action of $\mathbf{P G L}(2, \mathbf{Z} / 3)$ on the 4 points in $h$. This character is -1 only on the permutations (12) and $(12)(34)$, and it is a trivial matter to induce this to $\mathrm{S}_{4}$. It gives $3 \oplus 3^{\prime}$, where 3 and $3^{\prime}$ are the two irreducible 3 -dimensional representations of $\mathbf{P G L}(2, \mathbf{Z} / 3)$. Viewed 
now as representations of $\mathbf{G L}(2, \mathbf{Z} / 3), 3$ and $3^{\prime}$ are precisely the representations denoted $\mathrm{St}_{L}$ and det. $\mathrm{St}_{L}$ in [14 pp. 150-154], where $L=\mathbf{G L}(2, \mathbf{Z} / 3)$. We need to compute

$$
\operatorname{Ind}_{P\left(l_{1}, l_{2}\right)}^{G}(\psi)=\operatorname{Ind}_{P(h)}^{G}\left(\widehat{\operatorname{St}_{L}}\right)+\operatorname{Ind}_{P(h)}^{G}\left({\widehat{\operatorname{det} \cdot \mathrm{St}_{L}}}\right) .
$$

For this we need a little of the calculus of Deligne-Lusztig functors. First note that 14, p. 151]

$$
\begin{aligned}
\mathrm{R}_{T}^{L}(1) & =1+\mathrm{St}_{L}, \\
\mathrm{R}_{T}^{L}(\operatorname{det}) & =\operatorname{det}+\operatorname{det} \cdot \mathrm{St}_{L},
\end{aligned}
$$

where

$$
T=\left\{\left(\begin{array}{cc}
t_{1} & 0 \\
0 & t_{2}
\end{array}\right)\right\} \subset L=\mathbf{G L}(2, \mathbf{Z} / 3) .
$$

Note that $T$ is in a natural way an ( $F$-stable) maximal torus contained both in $B$, the Borel, and in the parabolic $P(h)$. Therefore we may repeatedly apply [38, prop. 2.6, p. 10] to get

$$
\begin{aligned}
\mathrm{R}_{T}^{G}(1) & =\operatorname{Ind}_{B}^{G}(1) \\
& =1+15 a+15 b+2 \cdot 24 a+81 a \\
& =\operatorname{Ind}_{P(h)}^{G}\left(\widehat{\mathrm{R}_{T}^{L}(1)}\right) \\
& =\operatorname{Ind}_{P(h)}^{G}(1)+\operatorname{Ind}_{P(h)}^{G}\left(\widehat{\operatorname{St}_{L}}\right) \\
& =1+15 b+24 a+\operatorname{Ind}_{P(h)}^{G}\left(\widehat{\operatorname{St}_{L}}\right)
\end{aligned}
$$

where we have used some results from the Atlas. This yields $\operatorname{Ind}_{P(h)}^{G}\left(\widehat{\mathrm{St}_{L}}\right)=15 a+$ $24 a+81 a$. Similarly,

$$
\begin{aligned}
\mathrm{R}_{T}^{G}(\mathrm{det}) & =\operatorname{Ind}_{B}^{G}(\widehat{\mathrm{det}}) \\
& =5 a+5 b+2 \cdot 30 a+45 a+45 b \\
& =\operatorname{Ind}_{P(h)}^{G}\left(\widehat{\mathrm{R}_{T}^{L}(\mathrm{det})}\right) \\
& =\operatorname{Ind}_{P(h)}^{G}(\widehat{\mathrm{det}})+\operatorname{Ind}_{P(h)}^{G}\left(\widehat{\mathrm{det} \cdot \mathrm{St}_{L}}\right) \\
& =40+120,
\end{aligned}
$$

where we have used the previously mentioned calculation of Springer. The only possibility to make the dimensions come out is

$$
\begin{aligned}
\operatorname{Ind}_{P(h)}^{G}(\widehat{\operatorname{det}}) & =5 a+5 b+30 a, \\
\operatorname{Ind}_{P(h)}^{G}\left(\widehat{\operatorname{det} \cdot \operatorname{St}_{L}}\right) & =30 a+45 a+45 b .
\end{aligned}
$$

Putting this all together gives part 2 of the proposition.

Step 3. $S^{1}=\operatorname{Ind}_{P(l)}^{G}(\varphi)$.

$P(l)$ is the stabilizer of a boundary component $D(l)$, and $\varphi$ gives the action on $\mathrm{H}^{2}(D(l), \mathbf{Q})$ (the local system $\varepsilon$ is trivial along $D(l)$ ). This action on the second homology of the boundary component is nontrivial on both factors of the decomposition $P(l)=U(l) \cdot L(l)$, so this is very far from the Harish-Chandra induction considered in the previous two cases. $P(l)$ consists of matrices

$$
\left(\begin{array}{cc|cc}
1 & u & v & s \\
0 & a & a s-b u & b \\
\hline 0 & 0 & 1 & 0 \\
0 & c & c s-d u & d
\end{array}\right) \quad \text { with } \quad\left(\begin{array}{cc}
a & b \\
c & d
\end{array}\right) \in L(l)=\mathbf{S L}(2, \mathbf{Z} / 3)
$$


Actually, the ones along the diagonal are really \pm 1 , but we are working in the projective group. We think of $D(l)$ as being the boundary component where the corresponding point in the closure of $\mathfrak{S}_{2}$ has the form

$$
\left(\begin{array}{cc}
i \infty & z \\
z & \tau
\end{array}\right) \text { with } z \in \mathbf{C}, \operatorname{Im}(\tau)>0
$$

and we think of the open part $D^{\circ}(l)$ as the quotient of the set of $(z, \tau)$ modulo the equivalence

$$
(z, \tau) \sim\left(\frac{z+u \tau+s}{c \tau+d}, \frac{a \tau+b}{c \tau+d}\right)
$$

for all

$$
\left(\begin{array}{ll}
a & b \\
c & d
\end{array}\right) \equiv\left(\begin{array}{ll}
1 & 0 \\
0 & 1
\end{array}\right) \bmod 3 \text { in } \mathbf{S L}(2, \mathbf{Z})
$$

and all $u \equiv s \equiv 0 \bmod 3$. In other words, we consider the group of matrices of the same shape as those of $P(l)$, but with integer entries, subject to the above conditions. The action is independent of the $v$-component. Also, it is rather clear from the above expression that the group $P(l)$ acts compatibly, via the projection $P(l) \rightarrow L(l)$, with the natural map to the corresponding modular curve $D(l) \rightarrow$ $M(l)$. We choose a basis of $\mathrm{H}^{2}(D(l), \mathbf{Q})$ consisting of

1) Any fiber of $D(l) \rightarrow M(l)$. As these are all homologous, the preceding remark shows that this class is invariant under $P(l)$.

2) The 9 universal sections of order 3 .

Therefore we have a decomposition $\varphi=1+\xi$, where $\xi$ gives the action on the points of order 3. The Atlas gives the decomposition $\operatorname{Ind}_{P(l)}^{G}(1)=1+15 a+24 a$, so we do the other part. The universal points of order 3 are easily identified in this picture as the classes of

$$
(z, \tau)=\left(\sigma_{m, n}(\tau), \tau\right)=(m \tau+n, \tau)
$$

where the integers $m, n$ are well-defined modulo 3 . The typical element of $P(l)$ will send this to

$$
\left(\begin{array}{c}
\frac{(m+u) \tau+(n+s)}{c \tau+d} \\
\frac{a \tau+b}{c \tau+d}
\end{array}\right)=\left(\begin{array}{c}
\sigma_{\mu, \nu}\left(\frac{a \tau+b}{c \tau+d}\right) \\
\frac{a \tau+b}{c \tau+d}
\end{array}\right)
$$

where

$$
\left(\begin{array}{c}
\mu \\
\nu
\end{array}\right)=\left(\begin{array}{c}
d(m+u)-c(n+s) \\
-b(m+u)+a(n+s)
\end{array}\right)
$$

We can identify the group of sections of order 3 with vectors $(m, n)$ in $\mathbf{F}_{3}^{2}$ and, where $P(l)$ acts on these vectors according to the rule $(m, n) \mapsto(\mu, \nu)$, where $(\mu, \nu)$ is given by the above expression. The representation $\xi$ is the associated permutation representation, and so $\xi(g)$ is simply the number of fixed points of $g \in P(l)$ acting on the $(m, n)$. The decomposition of this character was done on a computer with a MATHEMATICA program, using the character table from the Atlas, and the wellknown formula for the character of an induced representation. Also, we made use 
of the tables of Srinivasan (suitably corrected) in identifying the conjugacy classes with actual matrices. The result was

$$
\operatorname{Ind}_{P(l)}^{G}(\xi)=1+2 \cdot 15 a+20 a+2 \cdot 24 a+30 a+45 a+45 b+60 a+81 a .
$$

This gives part 3) of the proposition.

Remark. Consider any intermediate group $\Gamma_{2}(3) \subset \Gamma \subset \mathbf{S p}(4, \mathbf{Z})$. Because of the equality:

$$
\mathrm{H}^{i}(\Gamma, \mathbf{C})=\mathrm{H}^{i}\left(\Gamma_{2}(3), \mathbf{C}\right)^{\Gamma / \Gamma_{2}(3)},
$$

the results of this section allow the computation of the rational cohomology of the group $\Gamma$. Namely, the dimension will be the inner product of the characters

$$
\left\langle\mathrm{H}^{i}\left(\Gamma_{2}(3), \mathbf{C}\right), 1\right\rangle_{\Gamma / \Gamma_{2}(3)},
$$

and this is computable from the character table of the finite group $\operatorname{PSp}\left(4, \mathbf{F}_{3}\right)$.

\section{REFERENCES}

[1] Artin, M. and Mumford, D. Some elementary examples of unirational varieties which are not rational, Proc. London Math. Soc. (3) 25 (1972), 75 -95. MR 48:299

[2] Ash, A. Mumford, D. Rappoport, M. and Tai, Y. "Smooth compactifications of locally symmetric varieties", Math. Sci. Press, 1975. MR 56:15642

[3] Baily, W. L. and Borel, A. Compactification of arithmetic quotients of bounded symmetric domains, Ann. of Math. 84 (1966) 442 - 528. MR 35:6870

[4] Baker, H. F. "A locus with 25920 linear self-transformations", Cambridge Tracts in Mathematics and Mathematical Physics, no. 39, Cambridge University Press, 1946. MR 8:400b

[5] Borel, A. Stable and real cohomology of arithmetic groups, Ann. Sci. Ec. Norm. Sup. 7 (1974), $235-272=$ Collected Works of A. Borel, III, Springer - Verlag (1983), 315 - 352. (II). In: J. Hano et al. (ed.) Manifolds and Lie Groups (Notre Dame, IN, 1980; J. Hano et al., eds.), Progr. Math. 14, Birkhauser, 1981, pp. 21 - 55 = Collected Works of A. Borel, III, Springer - Verlag (1983), 650 - 684. MR 52:8338 MR 83h:22023 MR 85:01027c

[6] Borel, A., Carter, R., Curtis, C. W., Iwahori, N., Springer, T. A. and Steinberg, R. Seminar on Algebraic Groups and Related Finite Groups, Lecture Notes in Math. 131, Springer Verlag 1970. MR 41:3186

[7] Borel, A. and Serre, J. - P. Corners and arithmetic groups, Comment. Math. Helv. 48 (1973) $436-491$ = Collected Works of A. Borel, III, Springer - Verlag, 1983, 244 - 299. MR 52:8337 MR 85:01027c

[8] Borel, A. and Wallach, N. Continuous cohomology, discrete subgroups, and representations of reductive groups, Ann. of Math. Studies 94, Princeton U. Press, 1980. MR 83c:22018

[9] Burkhardt, H. Beiträge zur Theorie der hyperelliptischen Sigmafunctionen, Math. Ann. 32 (1888) 381 - 442; Grundzüge einer allgemeinen Systematik der hyperelliptischen Funktionen I. Ordnung. Nach Vorlesung von F. Klein, Math. Ann. 35, 1889, 198 - 296; Untersuchungen aus dem Gebiet der hyperelliptischen Modulfunctionen (I), Math. Ann. 36 (1890) 371 - 434; (II), Math. Ann. 38 (1891) 161 - 224; (III), Math. Ann. 41 (1893) 313 - 343; Ueber einen fundamentalen Satz der Lehre von den endlichen Gruppen linearer Substitutionen, Math. Ann. 41 (1893) 309 - 312.

[10] Conway, J. H., Curtis, R. T., Norton, S. P., Parker, R. A., Wilson, R. A. Atlas of finite groups, Oxford University Press, Oxford, 1985. MR 88g:20025

[11] de Jong, A. J., Shepherd-Barron, N. I. and van de Ven, A. On the Burkhardt quartic, Math. Ann. 286 (1990) 309 - 328. MR 91f:14038

[12] Deligne, P. Théorie de Hodge II, III, Publ. IHES 40 (1972), 5 - 57 and 44 (1975), 6 - 77. MR 58:16653a MR 58:16653b

[13] Deligne, P., Beilinson, A. A. and Bernstein, J. Faisceaux pervers, Astérisque 100 (1982). MR 86g:32015

[14] Digne, F., and Michel, J., Representations of Finite Groups of Lie Type, London Mathematical Society Student Texts 21, Cambridge University Press, 1991. MR 92g:20063 
[15] Durfee, Alan H. Intersection homology Betti numbers, Proc. Amer. Math. Soc. 123 (1995), 989-993. MR 95e:14014

[16] Faltings, G. and Chai, C. L. Degenerations of abelian varieties, Springer-Verlag, 1990. MR 92d:14036

[17] Franke, H. Hilbert modulflächen in Siegelscher modulvarietäten, Bonner Math. Schrft.

[18] Harder, G. A Gauss-Bonnet formula for discrete arithmetically defined groups, Ann. Sci. Éc. Norm. Sup. (4) 4 (1971) 409 - 455. MR 46:8255

[19] Hartshorne, R. Algebraic Geometry, Springer - Verlag, 1977. MR 57:3116

[20] Heidrich, H. and Knöller, F. W. Über die Fundamentalgruppen Siegelscher Modulvarietäten vom Grade 2, Manus. Math. 57 (1987) 249 - 262. MR 88e:11033

[21] Hoffman, J. W. The zeta function of Burkhardt's quartic, (1995) .

[22] Hoffman J. W. and Weintraub, S. H. Cohomology of the Siegel modular group of degree two and level four, Mem. Amer. Math. Soc., 133 (1998), no. 631. MR 98j:11039

[23] Hoffman J. W. and Weintraub, S. H. Four-dimensional symplectic geometry over the field with three elements, and a moduli space of abelian surfaces, in preparation.

[24] Hoffman J. W. and Weintraub, S. H. Cohomology of the boundary of Siegel modular varieties of degree two, with applications, in preparation.

[25] Howlett, R. B., and Lehrer, G. I., Induced cuspidal representations and generalized Hecke rings, Invent. Math. 58 (1980), 37 - 64. MR 81j:20017

[26] Hulek, K., Kahn, C. and Weintraub, S. H. Moduli spaces of abelian surfaces: Compactifications, degenerations and theta functions, Walter de Gruyter, Berlin, New York, (1993). MR 95e:14034

[27] Hunt, B., The geometry of some special arithmetic quotients of bounded symmetric domains, Lecture Notes in Math 1637, 1996. MR 98c:14033

[28] Hunt, B. and Weintraub, S. H. Janus-like algebraic varieties, J. Differential Geom. 39 (1994) 509 - 557. MR 95e:14026

[29] Igusa, J. -I. A desingularization problem in the theory of Siegel modular functions, Math. Ann. 168 (1967), 228 - 260. MR 36:1439

[30] Kazhdan, D. Connection of the dual space of a group and the structure of its closed subgroups, Funct. Anal. Appl. 1 (1967), 63 - 65. MR 35:288

[31] Kottwitz, R. Points on some Shimura varieties over finite fields, J. Amer. Math. Soc. 5 (1992), 373 - 444. MR 93a:11053

[32] Lee, R. and Weintraub, S. H. Cohomology of a Siegel modular variety of degree 2, Groups Acting on Manifolds (Boulder, CO, 1983; R. Schultz, ed.), Contemp. Math. 36 (1985), 433 488. MR 87g:11056

[33] Lee, R. and Weintraub, S. H. Cohomology of $\mathrm{Sp}_{4}(\mathbf{Z})$ and related groups and spaces, Topology 24 (1985), 391 - 410. MR 87b:11044

[34] Lee, R. and Weintraub, S. H. Topology of the Siegel spaces of degree two and their compactifications, Topology Proceedings 11 (1986), 115 - 175 . MR 89c:32071

[35] Lee, R. and Weintraub, S. H. The Siegel modular variety of degree two and level four: a report, in Arithmetic of Complex Manifolds, W. - P. Barth and H. Lange, eds., Lecture Notes in Mathematics 1399, Springer Verlag 1989. MR 90k:11061

[36] Lee, R. and Weintraub, S. H. The Siegel modular variety of degree two and level four, Mem. Amer. Math. Soc., 133 (1998), no. 631. MR 98i:57002

[37] Looijenga, E. $L^{2}$-cohomology of locally symmetric varieties, Compos. Math. 67 (1987), 3 20. MR 90a:32044

[38] Lusztig, G. Representations of Finite Chevalley Groups, Conf. Board Math. Sci. Regional Conf. Ser. Math. 39, Amer. Math. Soc., Providence, RI, 1978. MR 80f:20045

[39] MacPherson, R. and McConnell. M. Explicit reduction theory for Siegel modular threefolds, Invent. Math. 111 (1993), 575 - 625. MR 94a:32052

[40] Maschke, H. Über die lineare Gruppe der Borchardt'schen Moduln, Math. Ann. 31 (1887), 496 - 515; Aufstellung des vollen Formensystems einer quaternären Gruppe von 51840 lineare Substitutionen, Math. Ann. 33 (1889), 317 - 344.

[41] Namikawa, Y. "Toroidal compactifications of Siegel spaces", Springer Lecture Notes 812, 1980. MR 82a:32034

[42] Oda, T. and Schwermer, J. Mixed Hodge structures and automorphic forms on Siegel modular varieties of degree two, Math. Ann. 286 (1990), 481 - 509. MR 90m:11072 
[43] Ohsawa, T. On the $L^{2}$ cohomology of complex spaces, Math. Z., 209 (1992), 519 - 530. MR 93d:32063

[44] Saito, M. Mixed Hodge modules and applications. Proceeding of the International Congress of Mathematicians, Kyoto, Springer - Verlag (1991), 725 - 734. MR 93d:32059

[45] Sankaran, G. K. Fundamental group of locally symmetric varieties, Manus. Math. 90 (1996), 39 - 48. MR 97b:14017

[46] Saper, L. and Stern M. $L^{2}$-cohomology of arithmetic varieties. Proc. Nat. Acad. Sci. USA 84 (1987), 5515 - 5519. MR 89g:32052

[47] Satake, I. On the compactification of the Siegel space, J. Indian Math. Soc. 20 (1956), 259 281. MR 18:934c

[48] Serre, J.-P. Facteurs locaux des fonctions zêta des variétés algebriques (définitions et conjectures), Séminaire Delange - Pisot - Poitou (1969/70), Exposé 19, = Collected Papers II, 581 - 592. MR 53:5224 MR 89h:01009b

[49] Serre, J. -P. Motifs, Astérisque, 198 - 199 - 200 (1991), 333 - 349. MR 92m:14002

[50] Schwermer, J. On arithmetic quotients of the Siegel upper half space of degree two, Compositio Math., 58 (1986), 233 - 258. MR 87j:11040

[51] Shioda, T. On elliptic modular surfaces, J. Math. Soc. Japan, 24 (1972), 20 - 59. MR 55:2927

[52] Siegel, C. - L. Symplectic Geometry, Academic Press, New York, 1964. MR 29:1362

[53] Srinivasan, B. The characters of the finite symplectic group $\operatorname{Sp}(4, q)$, Trans. Am. Math. Soc. 131 (1963), 488 - 525. MR 36:3897

[54] van der Geer, G. Note on abelian schemes of level three, Math. Ann. 278 (1987), 401 - 408. MR 89a:14053

[55] Weintraub, S. H. Letter to Bruce Hunt, Aug. 16, 1987.

[56] Weissauer, R. Differentialformen zu Untergruppen der Siegelschen Modulgruppe zweiten Grades, J. Reine Angew. Math. 391 (1988), 100 - 156. MR 89i:32074

[57] Weissauer, R. On the cohomology of Siegel modular threefolds, In Arithmetic of Complex Manifolds, W. - P. Barth and H. Lange (Eds.), Lecture Notes in Math. 1399, Springer Verlag, 1989, 155 - 170. MR 91e:11051

[58] Weissauer, R. The Picard Group of Siegel modular threefolds, J. Reine Angew. Math. 430 (1992), 179 - 211. MR 94e:11052

[59] Yamazaki, T. On Siegel modular forms of degree two, Amer. J. Math. 98 (1976), 39 - 53. MR 53:8517

Department of Mathematics, Louisiana State University, Baton Rouge, Louisiana 70803

E-mail address: hoffman@math.1su.edu

Department of Mathematics, Louisiana State University, Baton Rouge, Louisiana 70803

E-mail address: weintr@math.lsu.edu 